\title{
REVIEWS
}

\section{Design, clinical translation and immunological response of biomaterials in regenerative medicine}

\author{
Kaitlyn Sadtler ${ }^{1 *}$, Anirudha Singh ${ }^{1,2 *}$, Matthew T. Wolf ${ }^{1}$, Xiaokun Wang ${ }^{1}$, Drew M. Pardoll ${ }^{3}$ \\ and Jennifer H. Elisseeff ${ }^{\prime}$
}

\begin{abstract}
The field of regenerative medicine aims to replace tissues lost as a consequence of disease, trauma or congenital abnormalities. Biomaterials serve as scaffolds for regenerative medicine to deliver cells, provide biological signals and physical support, and mobilize endogenous cells to repair tissues. Sophisticated chemistries are used to synthesize materials that mimic and modulate native tissue microenvironments, to replace form and to elucidate structure-function relationships of cell-material interactions. The therapeutic relevance of these biomaterial properties can only be studied after clinical translation, whereby key parameters for efficacy can be defined and then used for future design. In this Review, we present the development and translation of biomaterials for two tissue engineering targets, cartilage and cornea, both of which lack the ability to self-repair. Finally, looking to the future, we discuss the role of the immune system in regeneration and the potential for biomaterial scaffolds to modulate immune signalling to create a pro-regenerative environment.
\end{abstract}

'Translational Tissue Engineering Center, Departments of Biomedical Engineering and Wilmer Eye Institute, Johns Hopkins University, Baltimore, Maryland 21287, USA ${ }^{2}$ Department of Urology, Johns Hopkins University School of Medicine, Baltimore, Maryland 21287, USA ${ }^{3}$ Sidney Kimmel Comprehensive Cancer Center, Johns Hopkins University School of Medicine, Baltimore, Maryland 21231, USA.

*These authors contributed equally to this work.

Correspondence to J.E. jhe@jhu.edu

Article number: 16040 doi:10.1038/natrevmats.2016.40

Published online 7 Jun 2016
Disease, trauma and congenital defects lead to tissue loss and the necessity to replace missing form and function. Using materials to replace tissues dates back hundreds of years, starting with wooden or bony prosthetics for teeth and digits ${ }^{1}$. Surgeons then - and now - were also creatively exploring strategies in 'autograft' transplantation, which uses a person's own healthy tissues to replace injured ones ${ }^{2}$. Advances in allotransplantation permitted the restoration of form and function to entire tissues and organs harvested from donors. However, the constraints of cultivating extra tissue in an individual's body and the limited availability of donor tissues led to the advent of the field of tissue engineering. Now, using biomaterials (with knowledge of cellular biology) and molecular signalling, the de novo building of organ and limb replacements is given the collective term of regenerative medicine ${ }^{3}, 4$.

The three historical components of tissue engineering - cells, scaffolds and biochemical cues - are today often considered in isolation as a result of the technical and regulatory translational challenges of combining materials, cells and biologics into one therapeutic ${ }^{5}$. The cellular component of the system is the only viable or living portion that secretes and builds the tissue. This component can be endogenous cells from the surrounding environment that migrate into a scaffold or cells recruited from distant sites: for example, bone marrow-derived stem cells. Exogenous cells that can be delivered to a patient include autologous cells, which are expanded in culture or prepared immediately after extraction, or allogeneic cells harvested from healthy donors. Autologous or allogeneic cells can be further categorized as stem cells (adult or embryonic) or fully differentiated cells. The scaffold was originally considered to be primarily a $3 \mathrm{D}$ physical support for these cells to adhere to, proliferate, secrete extracellular matrix (ECM) and build tissue. However, biological cues in many forms can be incorporated into the scaffold to enhance cellular function and tissue production, which is an important focus of current research.

Although nearly all tissues in the body are targets for tissue engineering, some tissues, such as the liver, have a high capacity for regeneration, whereas others, such as cornea, lack the ability to self-repair. Cartilage also shows limited self-healing or self-repairing capability as a consequence of the dense and non-vascularized tissue matrix, which limits the access of necessary nutritional supplies and stem cells. In this Review, we describe the effective integration of cells, scaffolds and biochemical cues to engineer regenerative biomaterial scaffolds for clinical applications, focusing on strategies that could speed up translation, including the minimal 


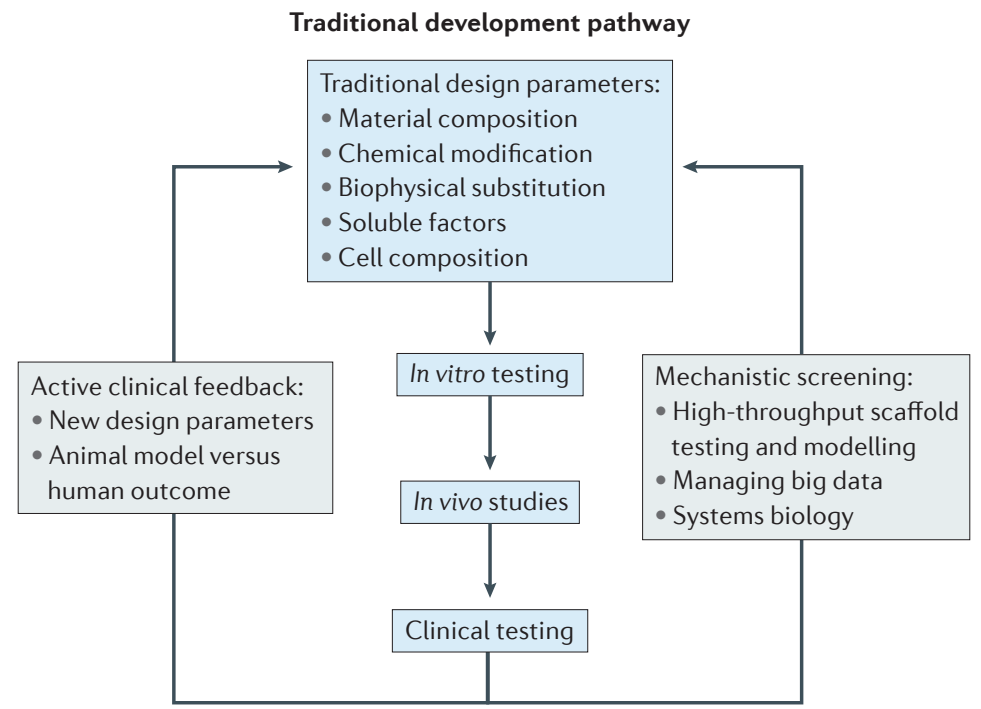

Figure 1 | New approaches to the traditional biomaterials development pathway. The paradigm for materials design and translation to the clinic involves material formulation, followed by in vitro testing of cytocompatibility and cellular responses to the material, in vivo testing of engraftment, biocompatibility and desired efficacy in animal models, and finally translation to the clinic for testing in human patients. Feedback from clinical testing and animal modelling can provide important information on the differences between a human response to a biomaterial and that of the model organism, and, most importantly, insights into the key therapeutic mechanisms that will dictate new design parameters for more relevant in vitro testing. In vitro analyses can reveal mechanisms by which the material interacts with various cell types and can guide subsequent chemical modification and processing. New techniques for analysing biological responses to materials are being developed to increase the efficiency of translation, including in vitro and in vivo arrays for high-throughput screening of complex biological outputs ${ }^{44,173}$.

manipulation of cells, the use of endogenous cells, and the simplicity in materials design and application. Strategies for cornea and cartilage repair and replacement, in which materials have already started to make substantial contributions to medicine, will be discussed.

\section{Design factors in regenerative biomaterials}

There are two approaches to engineering replacement tissues: in vitro cultivation and in vivo development ${ }^{6}$. In vitro cultivation, in simple static cultures or more complex bioreactors, aims to produce living tissues for transplantation that are ready for their desired biological function ${ }^{7,8}$. To generate functional tissues in vitro, biomaterials are generally required as a scaffold for cell attachment and growth, although there are some examples of cellular self-organization strategies that do not use materials, such as cell sheets and organoids ${ }^{9-11}$. In vitro cultivation - in cell culture media, or involving protein growth factors or small molecules - requires biological signals. These signals, which are chemical (for example, hydrophobicity) or physical (for example, shape and roughness) in nature, can be incorporated into the biomaterial scaffold to encourage tissue formation. Depending on the maturity of in vitro cultivated 'tissue', the biomaterial scaffold may or may not be present when the construct is implanted. Alternatively, the body can be used as an incubator for tissue development such that materials, cells or biological cues can be implanted alone or in combination to locally stimulate tissue growth ${ }^{12}$. Although less controlled than in vitro culture conditions, in vivo incubation and development provide innumerable biochemical and biophysical cues to support tissue growth. The traditional clinical development stretches from the basic materials design to in vitro testing, in vivo safety and efficacy studies, and finally to clinical testing (FIG. 1). The low probability of success and the long timelines of this traditional development pathway lead us to carefully consider how the process can be improved. Active feedback from clinical experience is required to understand the predictive capacity of in vivo studies and to delineate how preclinical models differ from people. Clinical testing also provides the potential to gain insight into the design parameters that are essential for therapeutic efficacy, thus improving future development.

Despite the complexity and signalling found in the local microenvironment in vivo, it is possible to consider using biomaterials alone to direct the tissue repair process. From the translational perspective, this approach to build new tissues has the advantages of more straightforward manufacturing and less burdensome regulatory pathways. Moreover, as biomaterials become more sophisticated with respect to biological cues, the potential for efficiently inducing in vivo tissue development and directing repair without adding cultured cells increases ${ }^{13}$. This shift in function of biomaterial scaffolds over the past 20 years, from passive physical structures to biointeractive structures (FIG. 2), changes the expectations of their performance and even, to a degree, the definition of a biomaterial. At the same time, this creates regulatory implications as the material gains biological and drug-like activity as a primary mechanism of action.

A balance between biomaterial simplicity and complexity must also be considered, because increasing scaffold complexity does not always lead to increased efficacy in rebuilding tissues in vivo and, most importantly, in the clinic. We propose to move beyond developing another polymer system that might provide incremental improvements in vitro to considering the whole in vivo system in which a tissue scaffold is placed. We are most interested in the complex crosstalk that occurs between distinct cell types and processes that can be influenced by materials to produce functional regeneration. It is clear that the design of biomaterials in regenerative medicine has reached a transformative point, where the link between in vitro engineering, in vivo preclinical and clinical outcomes is tenuous. New considerations, such as the relationship between the immune microenvironment, paracrine signalling and cellular recruitment, are now entering the discussion.

Biological cues. These cues come in many forms; however, stem cells are considered to be key components in the regeneration process as a result of their replicative power and multidifferentiation capacity for both in vitro tissue production and in vivo endogenous stimulation ${ }^{14}$. Biomaterials have been engineered with physical ${ }^{15,16}$, chemical $^{17,18}$ and biological ${ }^{19,20}$ cues to influence stem cell differentiation. With adult stem cells first and then 
with the more primitive embryonic stem cells, biomaterial functionalization could be tuned to stimulate and enhance differentiation and to produce functional tissues $^{18,21}$. Particularly with embryonic cells that require longer and more complex differentiation schemes, materials can have a central role in applications of in vitro culture control and differentiation, because they can integrate spatial and temporal control of biological cues ${ }^{21,22}$. Knowledge of developmental biology and signalling pathways becomes important so that such cues can be included in the scaffold material ${ }^{23,24}$. When the required biological signals are unknown, high-throughput combinatorial screening can help to identify candidate molecules to incorporate into a scaffold ${ }^{25}$.

Success using biomaterials to influence cell behaviour was traditionally defined by the efficiency of stem cell differentiation in vitro ${ }^{26,27}$. However, clinical translation of adult stem cells provides an important lesson on the potential mismatch of preclinical design parameters and therapeutic mechanisms of action in vivo and in clinical tests ${ }^{28}$. Because stem cells (alone without biomaterials) were tested in clinical applications, such as

\begin{tabular}{|l|l|}
\hline $\begin{array}{l}\text { Modern cancer vaccines and } \\
\text { immunotherapy }\end{array}$ & $\begin{array}{l}\text { Foreign body response } \\
\text { (1980s) Researchers begin to target } \\
\text { tumour-specific antigens and to } \\
\text { description of immune rejection } \\
\text { of synthetic biomaterials, } \\
\text { including neutrophil oxidation } \\
\text { to tune the immune response to } \\
\text { of environment and formation } \\
\text { of foreign-body giant cells }\end{array}$ \\
\end{tabular}

\begin{tabular}{|l|}
\hline Sutures and \\
stitches \\
Biting ants \\
used to \\
hold human \\
tissues \\
together
\end{tabular}

\begin{tabular}{|l|}
\hline Principles of immunology \\
(1700s-1800s) \\
Development of germ \\
theory and the first \\
vaccination
\end{tabular}

\begin{tabular}{|l|}
\hline Synthetic biomaterials \\
in ophthalmology \\
Intraocular lens of \\
poly(methyl methacrylate) \\
created for treating \\
cataracts \\
\hline
\end{tabular}

\section{Wound healing and} immunity (1980s-1990s) Immune cells, especially macrophages, are observed to have a role in wound healing

\section{Hydrogels for cell encapsulation} Tunable hydrogels and fibrous scaffolds created with controlled biological, chemical and physical properties to modulate tissue regeneration

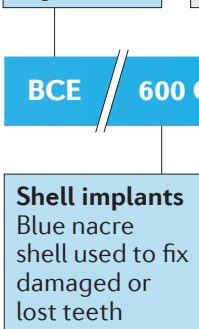

Immunology Biomaterials

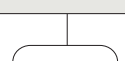

cataracts

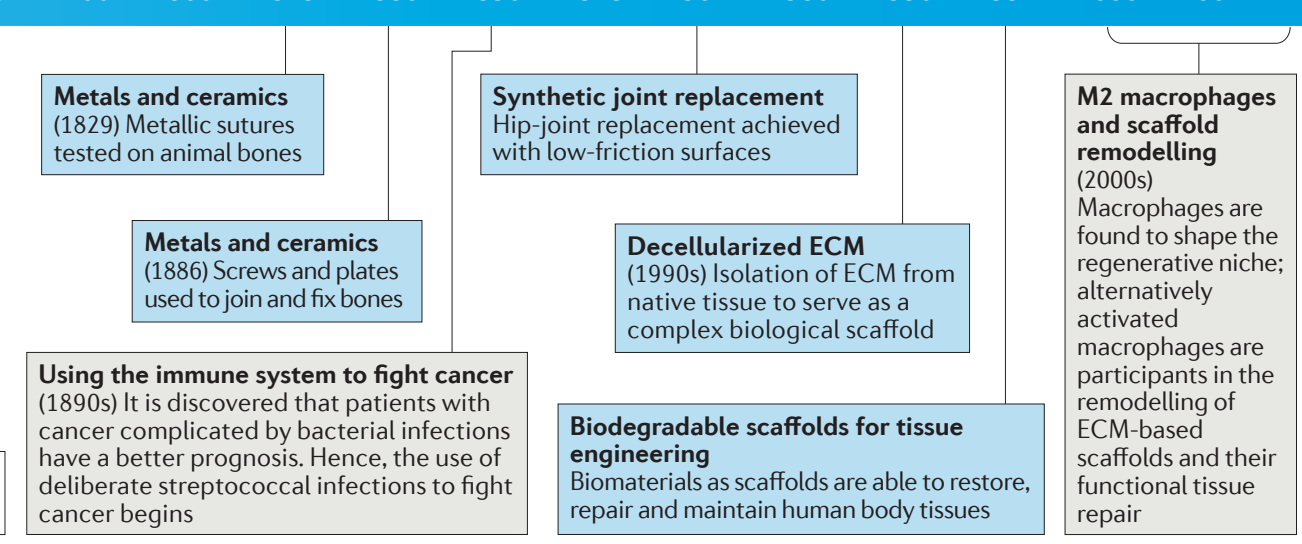

E
E
tiss
hy
tho
a

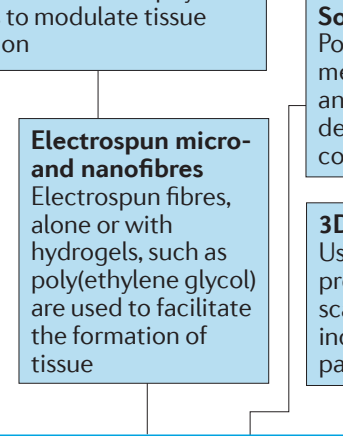

Soft contact lenses

Poly(hydroxyethyl

methacrylate) hydrogels and silicon hydrogels

developed for soft contact lenses

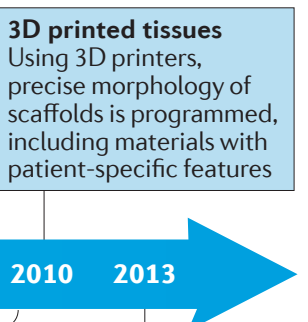

precise morphology o scaffolds is programmed, including materials with patient-specific features
Figure 2 | Evolving view of biomaterial interactions with the immune system. In Neolithic times, humans used materials such as the heads of large ants as sutures (to treat wounds) and shells (for dental prostheses) ${ }^{174}$. Over time, humans adapted materials, including metals and ceramics, to fix skeletal injuries ${ }^{175}$. Concurrently, principles of immunology were being developed, including early attempts at smallpox inoculation by Chinese scientists in 1549 (REF. 176) and vaccination by Edward Jenner in 1796 (REF. 177), as well as the confirmation of germ theory by Louis Pasteur in the late $1800 \mathrm{~s}^{178}$. The first implementation of immunoengineering was demonstrated with the use of commensal streptococcal infections to create an inflammatory antitumour response ${ }^{179}$. In the 1950s and 1960s, biomedical researchers took strides in applying synthetic materials to ocular and cartilage surfaces: for example, the Boston keratoprosthesis for treating cataracts $^{146}$, and the total knee replacement for severe arthritis and cartilage degradation ${ }^{180}$. In the 1980 s, the onset of modern immunoengineering arose with cancer immunotherapy and the identification of tumour-specific antigens $^{181}$. Around this time, James Anderson formally connected immune responses with biomaterials during the description of the canonical foreign-body response $\mathrm{e}^{33,34}$. In addition to responses to biomaterials, immune cells were connected with wound healing and tissue damage around the same time ${ }^{182,183}$. As immunotherapies became more directed and intricate, so did the design of biomaterials, including the formation of nanofibres ${ }^{121,130,149}$, hydrogels for cell encapsulation ${ }^{184,185}$ and improved synthetics for ocular applications ${ }^{186}$. In the 1990s and 2000s,

tissue-derived scaffolds, which were made by decellularizing native tissue to isolate the extracellular matrix $(\mathrm{ECM})^{69,187}$, began to be implemented as degradable, natural scaffolds that did not induce the large foreign-body response observed with their synthetic counterparts ${ }^{188}$. This anti-inflammatory immune response to ECM scaffolds was connected to a similar response associated with wound healing and tissue regeneration in lower vertebrates. Additionally, specific immune cell subpopulations, such as macrophages, eosinophils and T cells, were associated with tissue development and regeneration ${ }^{90-92}$. These advances in material integration and cooperation with the immune system of the patient were paralleled by engineering advances: for example, direct 3D printing of cell-laden scaffolds for the reconstruction of tissues and organs $s^{61,102}$. 
graft-versus-host disease, myocardial infarction and Crohn's disease ${ }^{29}$, it became apparent that the primary mode of action of the cells was not solely differentiation down a particular lineage but also cellular production of immunomodulatory factors, stimulation of proliferation and anti-apoptotic pathways in endogenous cells, and modulation of scarring processes ${ }^{30,31}$. Considering this, the success of biomaterial application to adult stem cells should focus on creating culture conditions to optimize the expression of these key factors and the delivery of cells in a desired phenotypic state. We are now starting to understand the importance of biological cues in the context of the immune system. When implanting a biomaterial scaffold, the immune system is the first responder to the foreign body. The biomaterial can in turn regulate the behaviour of immune cells, which have large and diverse secretomes. Targeting these early responders to the scaffold can influence the local microenvironment and attract key contributors in the subsequent regeneration process, such as stem cells and vascularization.

Biocompatibility. As biomaterials become sophisticated and integrate more complex biological signals, the concept of biocompatibility has evolved. Artificial hip implants and contact lenses are examples in orthopaedics and ophthalmology that have been used by millions of people since the mid-twentieth century. The metals and plastics that usually form these medical devices represent a historical view of biocompatibility, in which the material is passive and hardly interacts with surrounding tissue. Long-term use of synthetic implants can induce the formation of metal- or plastic-wear particles that may cause an inflammatory response that necessitates revision surgery ${ }^{32}$.

In the 1980s, James Anderson performed landmark studies that investigated the cellular and immune response to these passive materials ${ }^{33,34}$. More specifically, these studies applied a biomaterial implant cage system in rats, showing the acute and long-term inflammatory response to synthetic biomaterials and, hence, providing the basis for defining the foreign-body reaction to the biomaterials ${ }^{35-38}$. This work also emphasized the importance of macrophages in the chronic inflammatory response and their fusion to form foreign-body giant cells, the hallmark of the foreign-body reaction. Since these studies, our understanding of cell-material interactions has grown, and biomaterials have started to 'reach out' to the surrounding tissues and become biointeractive. An example is the addition of minerals to the stem of hip implants to promote bone ingrowth and, hence, to anchor and prevent movement ${ }^{39}$. Current thinking has now moved from desiring a thin fibrous capsule around an implant to favouring a seamless interface with surrounding tissue that could permit cellular migration into the biomaterial.

The local tissue environment has an important role in the immune response to a foreign antigen. For example, infections in the skin, heart and eye elicit very different immune responses. These tissue-specific responses are a result of many factors, including the phenotype of resident innate immune cells and the various receptors they express, the connection of the tissue to systemic factors (vascularity) and the ECM environment. As biomaterial scaffolds are a type of synthetic ECM, the body's response to a biomaterial not only depends on the physical properties of the biomaterial - for example, mechanical or architectural changes - but also on the local tissue environment. In other words, a biomaterial can induce varying responses based solely on tissue type and the location in which it is placed $^{40}$. We observed this phenomenon in the clinical testing of a hydrogel for soft-tissue reconstruction ${ }^{41}$. Soft-tissue damage is accompanied by the loss of tissue volume, which can be corrected by subcutaneous administration of biomaterials to fill the void left by the initial trauma or tissue removal. The translation of a composite biosynthetic material for soft-tissue-volume filling highlights the challenge of predicting the clinical compatibility of interactive materials in preclinical models and the importance of location or tissue-specific responses ${ }^{40-43}$.

Hydrogels composed of synthetic poly(ethylene glycol) (PEG) and doped with linear and crosslinked hyaluronic acid were injected into the abdominal subcutaneous space and crosslinked in situ using light ${ }^{41}$. The injections were performed 3 months before scheduled abdominoplasty, providing the unique opportunity for studying the local response to the biomaterial in humans. It was noted that the response to the implant varied depending on whether dermis, muscle or adipose tissue was adjacent to the implant, and, in addition, responses varied for different regions of the same implant. To further explore this concept, an array of tissue ECM spots was developed on which various cell types could be cultured and biological responses could be screened ${ }^{44}$. Combined with the proteomic profiles of each tissue, the approaches of gene ontology and systems biology can be applied to probe both mechanisms of local environmental influences and key factors in the therapeutic function of tissue-derived materials. Additional tissue and ECM arrays are being developed to model behaviour in vitro and to tease out mechanisms of repair ${ }^{45}$. If materials are to be designed to integrate with the body, the local context of the scaffold must be considered with respect to biocompatibility and also in terms of functional efficacy in regeneration.

Biomaterial scaffolds. Sophisticated biomaterial microenvironments have been synthesized in vitro to elucidate structure-function relationships between cells and materials. Synthetic polymers are useful for creating highly controlled cellular environments because structure and biological substitution can be tightly regulated ${ }^{13,17,18,46}$. Synthetic (and biological) materials can be further categorized into hydrogels and solid-like materials (such as non-water-soluble polymers) that are formulated into fibres ${ }^{47,48}$, sponges $^{49}$ or sheets ${ }^{50}$. In the case of synthetic hydrogels, such as PEG and polyacrylamides, mechanical properties (such as elastic and loss moduli) can be manipulated by simple changes in the crosslinking density that affect stem cell differentiation and phenotype ${ }^{18,51}$. The integration of biological signals in hydrogels has been accomplished by the simple mixing 
or doping of biopolymers, such as collagen or Matrigel, into the scaffold or even the cell-secreted $\mathrm{ECM}^{52,53}$. This mixing process does not take advantage of the chemical specificity of synthetics, and, as a result, several chemical functionalization strategies are available to covalently attach biologics or modify common biopolymers ${ }^{54,55}$. For example, hyaluronic acid hydrogels with a cell adhesive peptide (RGD) and a matrix-metalloproteinase-degradable peptide were created to probe how the cell-matrix interaction, through cell spreading and matrix degradability, regulates stem cell differentiation into specific lineages ${ }^{55}$. Recently, light-modulated hyaluronic acid hydrogels were used to facilitate the study of cell-ECM interactions in a high-throughput setup ${ }^{56}$.

Physical properties of 'solid' materials can be manipulated to influence cell behaviour by generating nanostructures such as fibres that can be aligned to further direct cells in the cases of nerve guidance tubes ${ }^{57,58}$ and ligament structures $^{59,60}$. 3D bioprinting introduces another dimension in the manipulation of scaffold and tissue architecture ${ }^{61-63}$. In addition to these physical properties, many synthetic scaffolds incorporate a biological element in the form of peptides, proteoglycans, proteins and complex mixtures. To avoid the need for multiple complex synthesis steps and hence increase the accessibility of the scaffold materials, click chemistry strategies have been developed to functionalize materials with signals such as cell adhesion peptides ${ }^{64,65}$. Even simple chemical functionalities, such as alcohols, acids, phosphates and amines, on synthetic polymers can influence biological responses, including stem cell differentiation ${ }^{17,18}$. Moreover, small molecules ${ }^{66-68}$ that can modulate cell behaviour open the door to embedding and delivering these signals that are complementary to the provision of protein and peptide-based cytokines, chemokines and growth factor options that are less stable.

Tissue-derived scaffolds. Instructing tissue development is complex and, as a result, the community has become increasingly interested in tissue-derived scaffolds rather than building new tissues from 'the ground up. The tissue ECM is itself a complex scaffold that is embedded with instructive signals that affect repair and regeneration. In fact, tissues and organs from animal and human sources have been used as a tool for reconstruction for centuries. The bladder, small intestine, submucosa, bone and dermis are examples of tissues with a long history of clinical use, and many other sources are being studied preclinically ${ }^{69-71}$. To engineer tissues and organs into transplantable materials, they are processed to kill cells and remove as much cellular debris as possible (namely, decellularization) ${ }^{69}$. Each unique tissue in the body presents different challenges in the removal of cells, and animal tissue introduces the added need to remove antigens that can cause rejection. The more extensively a tissue is processed, the more the physical and chemical composition change. The more minimal the processing of a tissue, the better it will serve as a scaffold for new tissue growth. Thus, a conundrum is presented in balancing minimal manipulation with biocompatibility.
One aspect of tissue-derived materials that concerns both materials scientists and regulatory agencies is the difficulty in understanding the composition (chemical and structural) of tissue-derived materials and thus what components are responsible for the therapeutic efficacy. Proteomics research is moving towards a better understanding of the tissue ECM composition ${ }^{72-74}$; however, questions still remain related to the basic understanding of tissue ECM. This gap in knowledge makes manufacturing consistency, whether from animal or human tissue sources, a challenge, particularly with respect to release criteria to ensure therapeutic efficacy. Nevertheless, these materials have been successfully implanted in millions of patients, have a strong safety record, and appear to promote tissue repair and reconstruction. As researchers continue to attempt to understand the mechanisms of action for these scaffolds ${ }^{44}$, the role of the immune system and the specific nature of the immune responses are becoming a primary focus of investigation. An overview of basic immune populations is shown in BOX 1. Furthermore, ECM-derived materials are now being integrated with synthetic materials, such as hydrogels, and are being sculpted with 3D printing strategies ${ }^{75}$; combining ECM with synthetics increases biocompatibility and cellular ingrowth in all of these cases ${ }^{76}$.

Acellular scaffolds implanted in tissue defects without cells are quickly invaded by endogenous cells that ultimately support tissue repair. However, it is not clear which cell types are attracted into the materials and which cells are responsible for tissue development. With a better understanding of how cells respond to materials, scaffolds can be designed to target specific cell types and optimize tissue regeneration even in synthetic materials. Early studies of biocompatibility showed that macrophage involvement is a key element in the long-term response to polymer biomaterials ${ }^{33,34,77,78}$. Traditionally ascribed roles in pro-inflammatory host defence processes, macrophages were thought of as protectors attempting (unsuccessfully) to remove biomaterials upon implantation, leading to frustrated phagocytosis, foreign-body giant cell formation and fibrotic capsule growth. By contrast, degradable biologically derived materials also recruited massive numbers of macrophages, but without developing a mature foreign-body reaction, which is characterized by the early infiltration of neutrophils followed by macrophages and frequently occurs with synthetic materials.

The concept of macrophage polarization states (or phenotypes) provides the framework for characterizing their diverse responses. Macrophage polarization was described as an extension of the Thelper $1\left(\mathrm{~T}_{\mathrm{H}} 1\right) /$ $\mathrm{T}_{\mathrm{H}} 2$ paradigm in $\mathrm{T}$ helper cells, in which, depending on their stimulus, macrophages can take a classical (M1) or alternative (M2) phenotype ${ }^{79}$. Further in vitro studies characterized the transcriptional and functional differences between these macrophage polarization states ${ }^{80}$. Macrophage phenotype correlated with tissue-specific host remodelling of biological materials, and less fibrosis - more specifically a greater ratio of $\mathrm{M} 2 / \mathrm{M} 1$ polarized macrophages - was found at sites of injury. Now, 
both synthetic and biological materials are constructed with macrophage modulation in mind $^{81,82}$. However, it is important to note that immune polarization is a complex continuum and that immune cells cannot be easily classified into distinct phenotypes ${ }^{83}$.

Conventionally, biomaterials researchers have preferred to categorize immune polarization into distinct states, such as M1 and M2 macrophages. A consequence of the diverse repertoire of challenges an organism may face is the need for a flexible response, and thus a simple M1 or M2 categorization is inherently incomplete. Instead of viewing immune polarization as a binary scale, with certain states being considered 'good' or 'bad' for regeneration, the immune response in wound healing and regeneration must be viewed as a delicately choreographed spatiotemporal pattern to yield successful tissue development ${ }^{84,85}$. This reflects the variability of macrophage phenotypes observed under many circumstances of resolution of immune responses, in which expression profiles and function do not fit a stereotyped polarization. A response that is appropriate for skin healing and regeneration may not be appropriate for ocular tissue or musculature, and the material must reflect these needs as opposed to only promoting a single immune phenotype ${ }^{86,87}$. Furthermore, the temporal pattern of the immune cells and their phenotypes is critical for the orchestration of the response and healing process. Similar to the early consideration of biomaterialsdirected stem cell differentiation, materials can now be designed to target and manipulate the complex pattern of the immune response.

\section{Box 1 | The immune system}

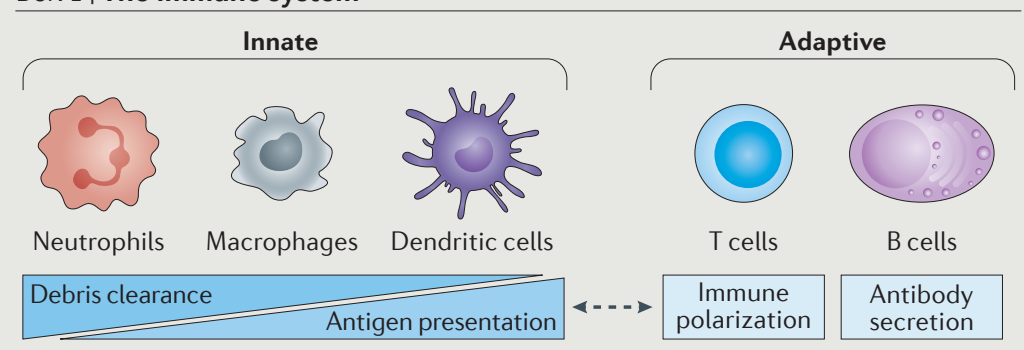

The immune system comprises both innate and adaptive compartments. The innate immune system responds quickly to a danger signal, is nonspecific and is mediated by polymorphonuclear cells (such as neutrophils, basophils and eosinophils), macrophages and dendritic cells. These cells kill and phagocytose pathogens or debris from wounding, then educate the adaptive immune system to form a specific response to the challenge that is presented. The adaptive immune system comprises cellular, T cell-mediated immunity and humoral, B cell-mediated immunity. B cells secrete antibodies that can target pathogens for degradation. $T$ cells can secrete cytokines that affect innate and adaptive immune cells. All cells of the immune system integrate signals from the surrounding environment to ensure that the response that is created is appropriate for the local tissue microenvironment. The response can be polarized to different categories of phenotypes by signals from the environment and secreted messengers; a T helper 1 $\left(T_{H} 1\right)$ response is associated with intracellular bacterial infections, a $T_{H} 17$ response induces secretion of antimicrobial peptides and defends against extracellular bacteria, a $\mathrm{T}_{\mathrm{H}} 2$ response is catered towards multicellular parasites and has a role in wound healing, and a regulatory $T\left(T_{\text {reg }}\right)$ cell response is a general immuno-inhibitory response that prevents overactivation of any of the other immune polarizations.

\section{The immune system in repair and regeneration}

Concurrent with research on the immunomodulatory role of tissue-derived materials, developmental and cell biologists are starting to probe the role of the immune system in repair and regeneration, thus creating a connection between the fields of regenerative materials, developmental biology and immunology (FIG. 3). In this section, we discuss the immune system in the context of biomaterials-mediated fibrotic responses, followed by a focus on macrophages and their role in scaffold remodelling and tissue regeneration in interactive biological scaffolds.

From the perspective of developmental biology, cells of both the innate and adaptive immune system have a role in the regeneration process. Immune cells signal through various secreted and cell surface proteins that define and dictate polarization phenotype, permit communication between the different immune cell types, and influence tissue development and regeneration by stimulating the surrounding stem and progenitor cells. More specifically, type 1 immune polarization, which is a pro-inflammatory phenotype, is driven by $\mathrm{T}_{\mathrm{H}} 1$ cells from the adaptive immune system. $\mathrm{T}_{\mathrm{H}} 1$ cells are maintained by interleukin-12 (IL-12) and produce interferon- $\gamma($ IFN $\gamma)$ as their canonical cytokine. They also produce M1 macrophages from the innate immune system expressing nitric oxide synthase (iNOS; also known as NOS2) and tumour necrosis factor (TNF) in response to the stimulation of Toll-like receptors (TLRs) or other intracellular pattern recognition receptors, together with IFN $\gamma$ produced by $\mathrm{T}_{\mathrm{H}} 1$ cells. $\mathrm{T}_{\mathrm{H}} 1$ and $\mathrm{M} 1$ responses are key elements to viral and intracellular bacterial infections, many of which can infect macrophages themselves.

Type 2 immune polarization, which is important in the response to multicellular parasites and wound healing, is characterized by $\mathrm{T}_{\mathrm{H}} 2$ cells expressing cytokines such as IL- 4 and IL-13, and M2 macrophages driven by these cytokines. M2 macrophages repair tissue destruction caused by the immune response to tissue infection and produce epidermal growth factor receptor (EGFR) ligands, vascular endothelial growth factors (VEGFs), transforming growth factor- $\beta$ (TGF $\beta$ ) and secrete specific pleiotropic mediators such as the cysteine-rich protein FIZZ1 (also known as resistin-like- $\alpha)^{83,88,89}$. M1 and M2 macrophage polarization can occur independently of T-cell responses but is strongly enhanced by $\mathrm{T}_{\mathrm{H}} 1$ and $\mathrm{T}_{\mathrm{H}} 2$ involvement, respectively. The two cell types often work synergistically, with macrophages receiving early signals from the environment and pathogens that cause a small M1 or M2 polarization, presenting antigens to and further inducing $\mathrm{T}$ cells towards the corresponding $\mathrm{T}_{\mathrm{H}} 1$ or $\mathrm{T}_{\mathrm{H}} 2$ lineage, which can in turn promote a more robust M1 and M2 phenotype in a feedforward loop. The initial priming of naive $\mathrm{T}$ cells requires dendritic cells, which can also polarize $\mathrm{T}$ cells towards $\mathrm{T}_{\mathrm{H}} 1$ or $\mathrm{T}_{\mathrm{H}} 2$ differentiation depending on their production of selected cytokines (more specifically, IL-12 for $\mathrm{T}_{\mathrm{H}} 1$ ).

As with a response to a foreign body, such as a pathogen, the immune system recognizes and polarizes its response to biomaterials that are used in tissue 
engineering and regenerative medicine. For example, macrophages are responsible for ECM remodelling and debris clearance, but they also secrete chemotactic signals that act to recruit other immune cells and act on surrounding parenchymal cells, including stem cells, to influence repair. Ablation of macrophages from the limb bud of a salamander using clodronate liposomes inhibits normal limb regeneration ${ }^{90}$. Even after the formation of a blastema in a later-stage regenerating limb, depletion of macrophages results in severely hindered limb growth and differentiation, and is accompanied by drastic changes in the immune microenvironment of the limb bud and increased collagen deposition.

The critical role of immune signals is exemplified in recent research on muscle repair. For example, the immune signalling protein IL-4 was required in murine muscle repair after cardiotoxin damage ${ }^{91}$. The IL-4 in this model was primarily secreted by eosinophils followed by monocytes. In the Il4ra-knockout mouse, muscle regeneration is severely inhibited, and muscle precursors are shifted to a pro-adipogenic state, which induces ectopic adipogenesis in the wound site. Additionally, in a volumetric muscle loss model, researchers have demonstrated a reliance on signals from the adaptive immune system, specifically $\mathrm{T}_{\mathrm{H}} 2$ cells, to determine the regenerative fate of ECM scaffolds $^{92}$. As previously described, an M2 macrophage phenotype was associated with an improved outcome of the remodelling of ECM scaffolds. Without $\mathrm{T}_{\mathrm{H}} 2$ cells present, the macrophages lose their pro-regenerative phenotype, which is also associated with IL-4 signalling, thereby defining them as pro-regenerative M(IL-4) macrophages. IL-4, specifically mediated by $\mathrm{T}_{\mathrm{H}} 2$ cells, was a critical determinant in fibro/adipogenic lineage commitment and biomaterial-mediated muscle regeneration.

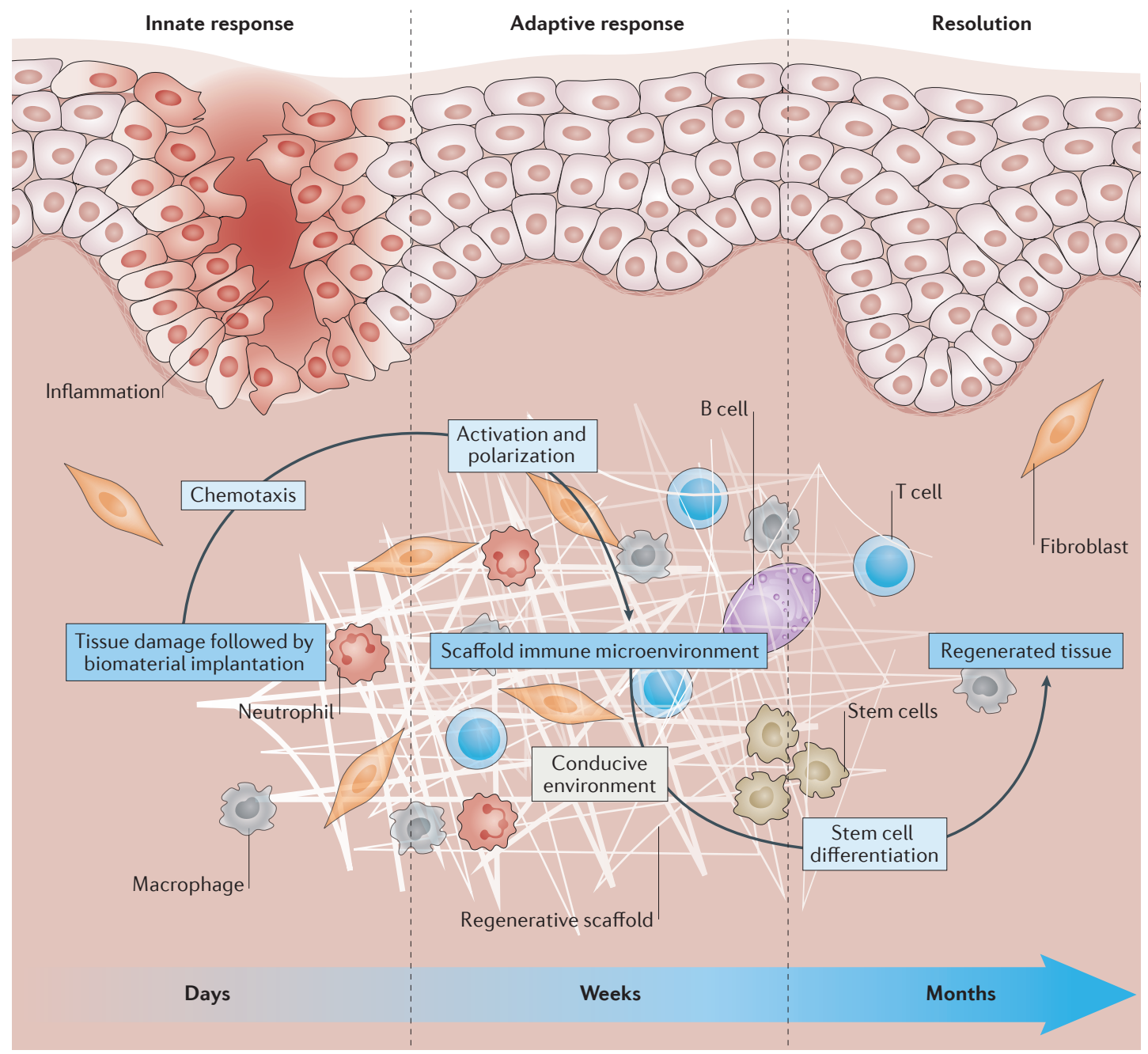

Figure 3 | Regenerative immunology and the associated role of biomaterials. Signals associated with injury (and the implantation of a scaffold) first bring innate immune cells to the region and activate local cells; this is followed by activation and polarization of adaptive immune cells. Depending on the phenotype of these immune cells, their interaction with other cell types, such as stem cells, and cues from the local microenvironment (including the biomaterial scaffold), either tissue resembling the original host tissue or more fibrous scar tissue can form. Formation of a pro-regenerative immune microenvironment supports the development of proper replacement tissue; however, unbalanced activation of the immune response can produce fibrosis or damaging inflammation. 

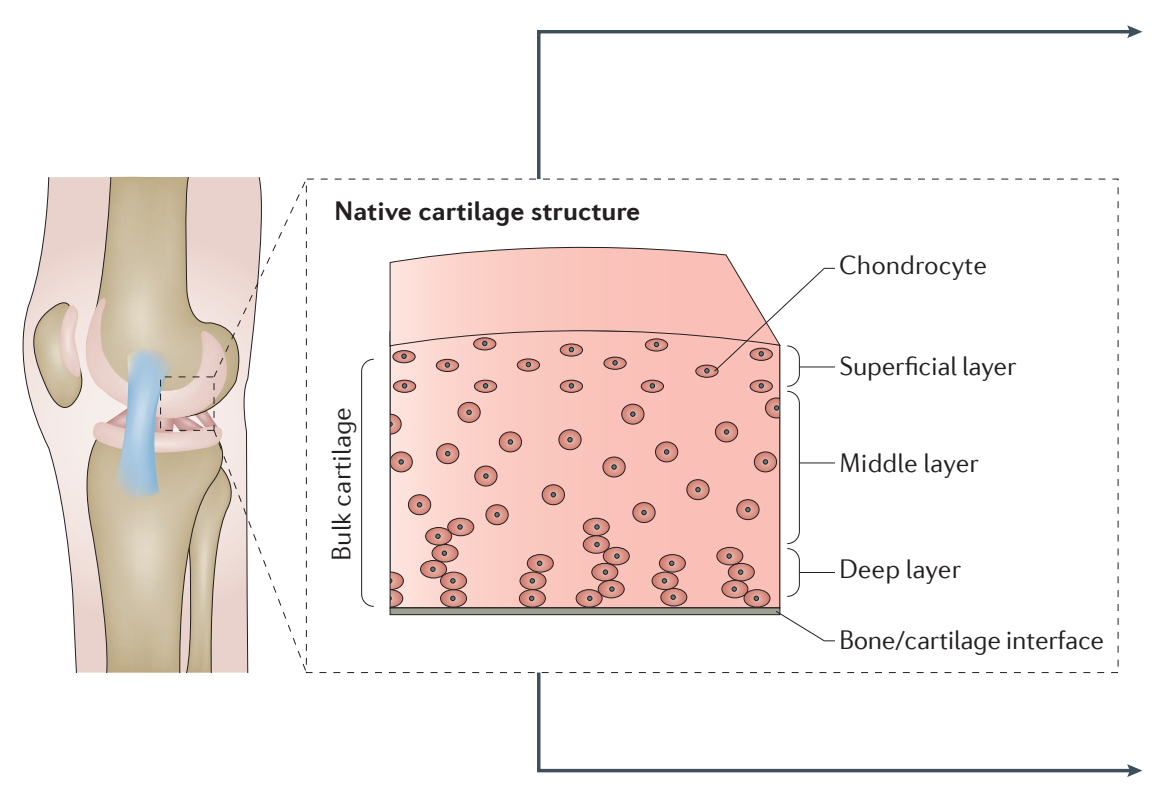

Polymer engineering of cartilage surface/fluid interface

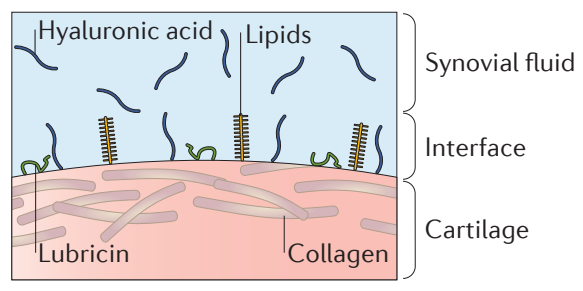

Materials for substituting bulk cartilage engineering

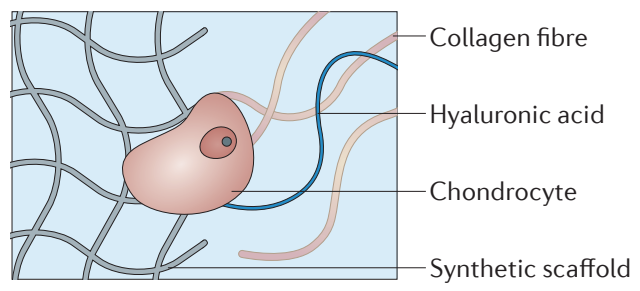

Figure 4 | Native cartilage structure and target tissues for engineering. Trauma or diseases, such as osteoarthritis, lead to the loss of cartilage that lines the surface of articulating joints. Treatment for cartilage damage can target the surface (cartilage/fluid interface) and/or the stromal tissue (bulk cartilage) to replace the lubrication and biomechanical load-bearing functions, respectively. The cartilage surface has molecules that can interact specifically with the synovial fluid, such as lubricin, lipids and hyaluronic acid. The bulk articular cartilage, which primarily comprises type II collagen and aggrecans, is avascular, with chondrocytes interspersed in the dense extracellular matrix.

In the same way that IL- 4 has been associated with myotube fusion and the formation of a more mature muscle tissue, it can also promote the formation of fused macrophages or foreign-body giant cells ${ }^{93}$. In this case, the immune signal that is an important positive signal for mature muscle formation has a negative effect on responses to non-degradable biomaterials. Studies in cardiac muscle repair also shed light on the complexity of the immune system and its impact on healing, whereby specific immune cell recruitment and activation lead to either successful healing or inflammatory pathologies ${ }^{94}$. Neutrophil infiltration, a common early innate immune response, is associated with a poor prognosis in cardiac tissue healing. Several categories of monocytes, which follow the neutrophils, were defined on the basis of their roles in cardiac tissue healing and regeneration. Tissue-resident monocytes are thought to be crucial for the clearing of dead cell debris and remodelling of the wounded cardiac tissue without triggering a large inflammatory response. However, these cells are also associated with autoimmune disease, highlighting the delicate balance that must be navigated.

The regenerative outcomes of both local and systemic immune profiles are now also being considered at the clinical level. For example, macrophages, T cells and $\mathrm{B}$ cells were found to be required for bone repair ${ }^{95,96}$, but differentiated $\mathrm{CD}^{+} \mathrm{T}$ cells negatively correlated with repair ${ }^{97}$. At the systemic level, specific blood immune signatures were correlated with fast or delayed surgical recovery ${ }^{98}$. In summary, the application of a biomaterial will increase the number and type of immune cells that migrate to a traumatic wound or injury site. The recruitment of these immune cells also relies on the tissue location in which the material is implanted, the presence or absence of trauma, and the physical and biological properties of the biomaterial present (FIG. 3). The biomaterial scaffold and surrounding tissue creates a scaffold immune microenvironment, which is characterized by the induction of immune polarization and activation towards different phenotypes (such as $\mathrm{T}_{\mathrm{H}} 1$ and $\mathrm{T}_{\mathrm{H}} 2$ cells) and the secretion of related cytokines. These immune cells can then influence the recruitment and/or differentiation of stem and progenitor cells in the scaffold, ultimately determining the regenerative outcome. We term this process biomaterials-directed regenerative immunology.

\section{Translational biomaterials}

Although in vitro and preclinical models can provide important information on safety and some insight into efficacy, combining this learning with the analysis of clinical trials provides a more comprehensive picture of relevant design parameters to optimize repair and regeneration in humans (FIG. 1). Highly controlled structure-function studies can give insight into cell-biomaterial responses and are necessary for the field ${ }^{99,100}$; however, the correlation of these results with therapeutic efficacy is far from clear, and the connection of in vitro and small animal preclinical model outcomes and their prediction of regeneration in people are uncharted. Clinical examples of regenerative biomaterial applications help to underscore the challenge 


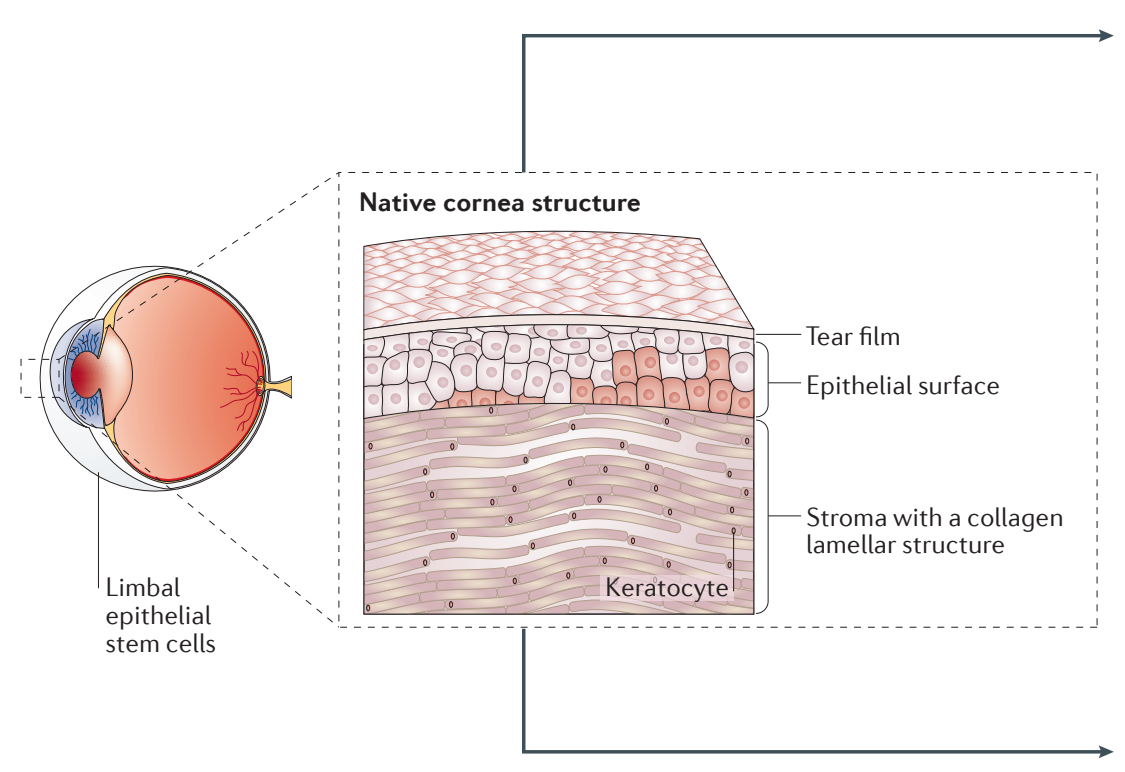

Epithelial surface of the cornea

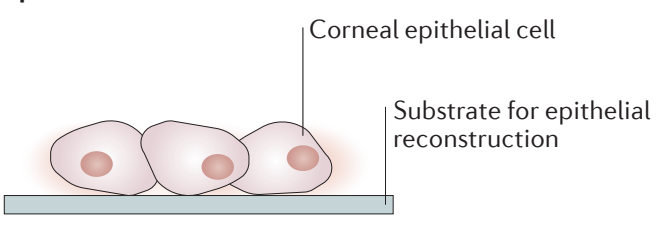

Materials for substituting bulk stroma tissue

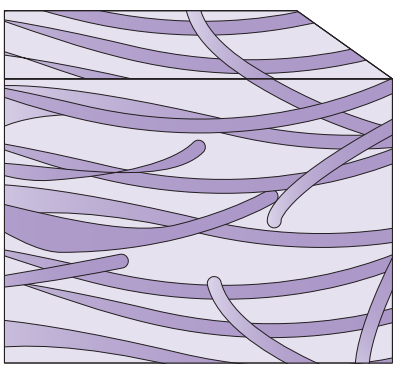

Figure 5 | Native cornea structure and target tissues for engineering. The human cornea is a complex tissue composed of several layers. Tissue engineered corneas mostly target the anterior parts, including the corneal epithelium and the stroma. The epithelium provides protection from the environment and supports lubrication of the eye. The stromal tissue is composed of highly organized collagen lamellar structures. This organization provides the cornea with its strength and clarity. Ideally, tissue engineered corneas would have similar strength and clarity to the stromal tissue and allow rapid re-epithelialization of the surface.

in defining mechanisms of action, design parameters and the importance of integrating translational experience in future materials development. In this section, we introduce translational biomaterials in the fields of orthopaedics and ophthalmology, focusing on cartilage and cornea tissue regeneration. Although seemingly disparate fields and tissues, there are actually several similarities in the structure and regenerative challenges (FIGS 4-7). Both the articular cartilage and the cornea are avascular tissues that have a dense, highly organized ECM, with cells interspersed at a relatively low density (FIG. 4,5). The complex organization of the ECM is responsible for the mechanical properties and specific functions (a protective clear cornea and low-friction cartilage) of the tissues. Both tissues suffer from an inability to self-repair and the predilection to form troublesome scarring when damaged, which reduces vision in the cornea and creates a weak fibrocartilage in the cartilage, thus impairing function. Interestingly, synthetic materials have a long history in the fields of orthopaedics and ophthalmology, which may serve as a barrier to the development of new and different materials for therapeutic or regenerative approaches for reconstruction. By comparison, fields with no existing materials options have been able to leapfrog development and test biological materials early: for example, for the replacement of trachea ${ }^{101,102}$.

Similarities extend beyond the ECM to the somewhat immune-privileged nature of the tissues and to cells that inhabit cartilage and cornea tissue - more specifically, chondrocytes and keratocytes. These cells are generally considered to be quiescent or to have low metabolic activity, and tend towards a fibroblastic phenotype when activated or isolated in culture ${ }^{103,104}$. Furthermore, these tissues live in complex, interconnected environments the articular joint and the ocular surface - that must be taken into account during therapeutic interventions. Ultimately, several biological and structural attributes converge to make articular cartilage and cornea tissues particularly challenging to regenerate. In the following sections, we review some approaches for regenerative materials design and cover the few examples of clinical experiences.

\section{Regenerative materials for cartilage repair}

Articular cartilage lines the surfaces of moving joints in the body, with the primary physical function of providing a low-friction surface for painless movement (FIC. 4). The loss of cartilage is associated with osteoarthritis and leads to pain and morbidity that affects millions of people, and its effect will continue to grow with the ageing population, making cartilage repair a valuable clinical target. The unique ECM structure of type II collagen and complex aggregating proteoglycans provide the tensile and compressive properties, respectively, with the crosslinked collagen fibres entrapping the hydrophilic proteoglycans. Until recently, the shear low-friction characteristics of the tissue were largely overlooked ${ }^{105}$. However, the surface of healthy articular cartilage contains proteins and a physical structure that interacts synergistically with components of the synovial fluid, which lubricates the surface to reduce friction in shear movements ${ }^{106}$. Thus, regenerative 
strategies and materials should promote recapitulation of both the cartilage tissue surface and bulk properties. Following the development paradigm of FIG. 1, the first phase of biomaterial application to cartilage encompasses in vitro cultivation and the evaluation of cartilage growth in the scaffold using either chondrocytes or mesenchymal stem cells $s^{50,107}$. The second phase of scaffold testing encompasses preclinical animal models, including small animal (rodent), rabbit and larger animal (goat or sheep) models ${ }^{108,109}$. The lack of predictive preclinical models has hampered the field; therefore, the feedback from clinical translation is crucial for better scaffold design in the future.

Articular cartilage function can be efficiently replaced clinically by artificial plastic and metal implants that first gained traction in the $1960 \mathrm{~s}^{110}$. In fact, this history of successful clinical use may have actually hindered the development and translation of more biological materials or strategies. These artificial implants have a limited lifetime, are subject to wear-particle formation and associated inflammation and do not completely replicate normal joint function, leading physicians and patients to search for alternatives. To this end, in the 1980s, autologous chondrocyte transplantation (ACI) was developed ${ }^{111,112}$. In this procedure, chondrocytes are isolated from a biopsy taken from the patient after which the cells are expanded in culture before re-injection into the cartilage defect. Although some patients, particularly of a younger age, benefit from ACI, clinical studies that compare this process with microfracture - a process that involves the stimulation of the underlying bone marrow - have shown more equivocal results with little added benefit in healing ${ }^{113,114}$. These disappointing cell therapy results may be explained by the fibroblastic shift of the chondrocyte cells in vitro ${ }^{103}$, challenges in maintaining cell viability and localization in the defect, which all combine to impede their ability to produce high-quality cartilage. The ACI approach also does not consider the importance of other cell types in the cartilage healing process.

Biomaterials can address some of the challenges in restoring cartilage and, ultimately, articular joint function (FIG. 6). When rebuilding cartilage tissue structure and function, the clinical need can be broadly categorized into two areas: replacing the bulk mechanical load-bearing function and providing a low-friction surface for painless movement. Thus, treatment modalities can be categorized into tissue and biomaterial surface modifications that enable interactions with components of the synovial fluid and increase lubrication (FIG. 6a); the development of biomaterials that mimic the bulk mechanical properties of native cartilage tissue (FIG. 6b); and the design of materials that modulate the wound healing process and promote new tissue development (FIG. 6c).

In normal tissue, the cartilage surface is organized with the ECM proteins aligned parallel to the surface and includes proteins such as lubricin, which binds hyaluronic acid in the synovial fluid. This interaction of the cartilage tissue surface with the synovial fluid creates a synergy that results in low-friction movement and lubrication. This lubrication is lost with tissue damage and arthritis, and is not found in repair tissue.
Enhancing lubrication of the articular surface can be addressed by modifying the synovial fluid compartment or the cartilage surface that interfaces with the synovial fluid. Polymeric supplementation of the synovial fluid compartment focuses on biopolymers, such as hyaluronic acid, charged polymers and large molecular brush structures. As these molecules will ideally work at the tissue interface to enhance lubrication, they can be covalently immobilized to cartilage. Recombinant lubricin proteins, and their mimics, are bound to the surface layer of cartilage and serve to interface with hyaluronic acid in the synovial fluid so that the lubricating polymer is fixed in the position needed to enhance lubrication. This native interface can be recreated with synthetic polymers that bind non-covalently to the tissue and synovial components using peptides ${ }^{115-118}$.

As cartilage is a largely avascular tissue with limited growth capabilities, biomaterials targeting bulk tissue engineering have been an important goal for regenerative medicine therapeutics. Several biomaterial scaffolds have been designed to enhance the chondrocyte cell delivery described earlier along with stem cell delivery. For example, chondrocytes were cultured on biosynthetic membranes to help to maintain cell phenotype during culture and to improve transplantation efficiency in a defect ${ }^{114}$. Cells have also been delivered in synthetic hollow microspheres ${ }^{119}$, and clinical studies have even used a biosynthetic adhesive to target and maintain cells in a desired location ${ }^{120}$. Cells can also be cultured on 3D scaffolds, in which case, they proliferate less but start to secrete ECM components (such as collagens and proteoglycans) and rebuild cartilage tissue $^{121}$. The primary therapeutic challenge with cartilage engineered in vitro is integration of the new tissue with native host tissue. Several integration strategies and adhesive materials have been developed to bridge this gap ${ }^{122}$. As an alternative, the interface of the engineered tissue and scaffold can be degraded to stimulate healing and integration with the host tissue in vivo. Polymeric biological adhesives can also provide both a physical bridge and biological cues to support tissue growth at the cartilage interface ${ }^{114,123}$.

Delivering cells, with or without the use of biomaterials, is costly and difficult. Thus, translational research has shifted from cell therapies to cell-biomaterial combination therapies and, finally, to materials alone. From a design perspective, materials that mimic the physical properties of cartilage tissue are a logical starting point. Biomaterial scaffolds have been designed to mechanically match native cartilage tissue using nanofibres and moulded microsphere building blocks, along with techniques of fabric weaving and 3D printing. These mechanically robust materials are implanted alone or can be seeded with cells (FIG. 6b). Alternatively, from a biological perspective, softer materials support chondrogenic differentiation of stem cells, whereas stiffer materials support osteogenic differentiation ${ }^{51}$ and fibroblastic scar growth. As a result, instead of mimicking mature cartilage properties, the material should be soft to create an environment reminiscent of early development that supports chondrogenesis. Hydrogels - water insoluble crosslinked 
Hyaluronic acid binding and lubrication

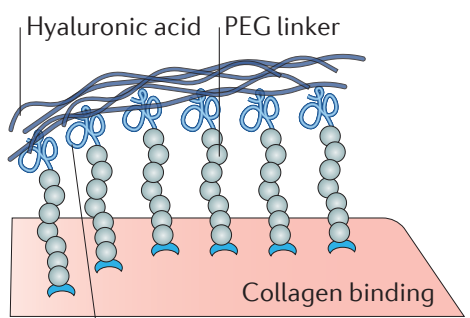

Hyaluronic acid-binding peptide
Cartilage surface lubrication

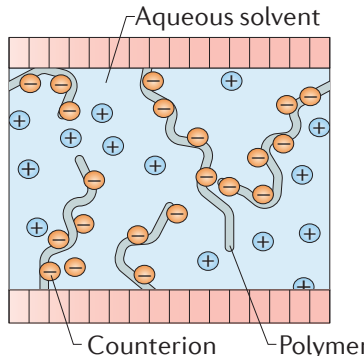

Synthetic mimic of lubricin to enhance lubrication

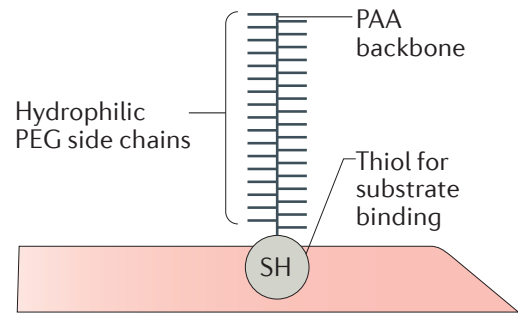

Recombinant lubricin to rebuild diseased interface

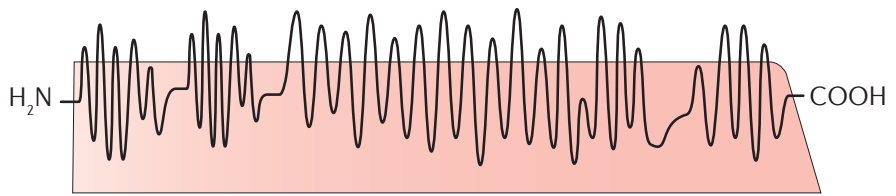

\section{Synthetic lubrication}

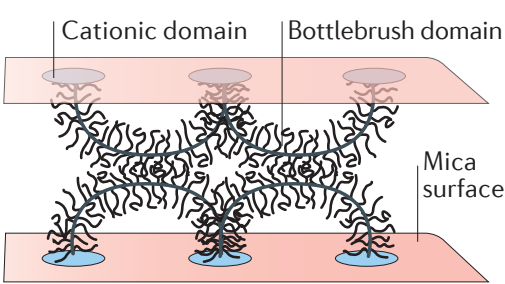

\section{b Biomaterials mimicking native tissue}

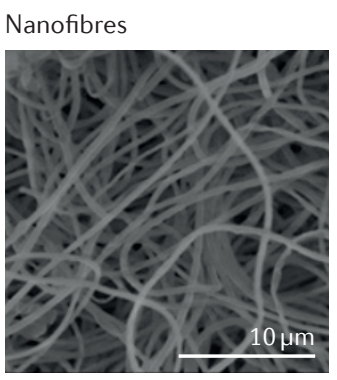

Woven scaffold

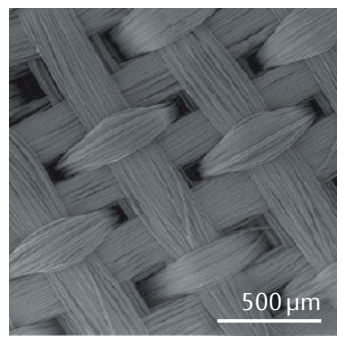

Microspheres and fibres

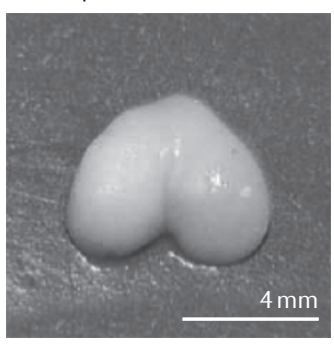

3D printed scaffold

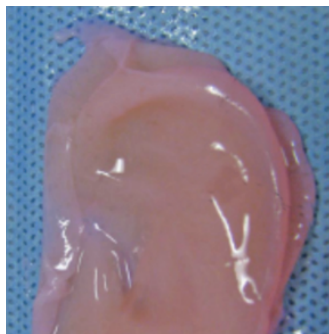

C Biomaterials that direct wound healing

Proteoglycan PEG hydrogel
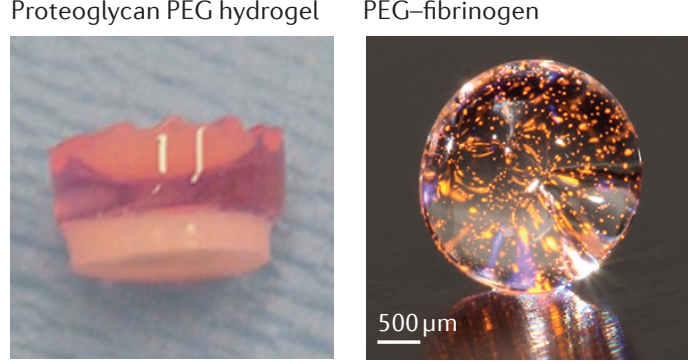

Peptide hydrogel

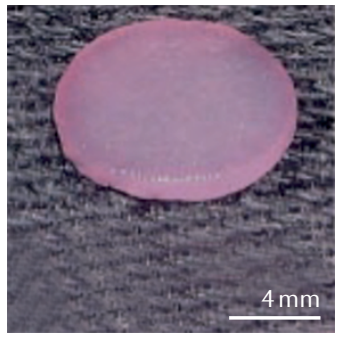

Multilayered chitosan hydrogel
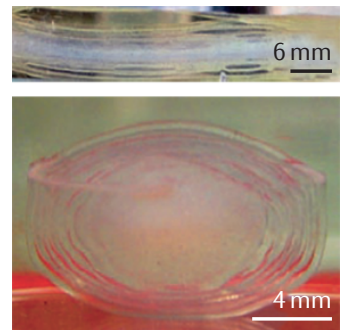

Figure 6 | Surface modifications and tissue engineering of bulk cartilage. a | Surface modification targets the surface of the cartilage/fluid interface and the constituents of synovial fluid. Hyaluronic acid injections are used clinically today to lubricate joints, and enhancements to target and bind hyaluronic acid to the tissue surface (mimicking the function of lubricin) using synthetic binding complexes are under development ${ }^{118}$. By modelling the lubrication chemistry of the joint, researchers have been able to describe the interactions between charged molecules and water that create the unique low-friction tissue surface ${ }^{106}$. Recombinant proteins ${ }^{189,190}$ and synthetic mimics ${ }^{115,116}$ have thus been designed to recreate the native tissue/synovial fluid interface. $\mathbf{b}$ |Biomaterials can be formulated into different physical forms, such as nanofibres ${ }^{121}$, microspheres ${ }^{119}$, woven scaffolds ${ }^{48}$ and intricate 3D printed materials ${ }^{63}$, to mimic cartilage properties and to support cell seeding and the development of bulk cartilage. c|Softer hydrogel materials, including proteoglycans ${ }^{127}$, fibrinogen ${ }^{46,129}$, synthetic peptides ${ }^{191}$ and chitosan ${ }^{124}$, provide an alternative approach to cartilage repair by working to activate endogenous stem cells after procedures, such as microfracture or combination, with intra-operative biologics to promote wound healing. PAA, poly(acrylic acid); PEG, poly(ethylene glycol). 
hydrophilic polymers - are soft materials that can serve as a scaffold for cartilage repair and can even be organized into more complex multilayer gels ${ }^{124}$. Several hydrogel formulations have progressed to clinical testing for filling cartilage defects, including PEG hydrogels in combination with a tissue adhesive ${ }^{125,126}$, chondroitin sulfate ${ }^{127}$, chitosan $^{128}$, PEG-fibrinogen ${ }^{129}$ and hyaluronic acid ${ }^{54}$. These hydrogels are applied without cells and implanted in conjunction with microfracture surgery that induces bleeding and mobilizes endogenous cells. Although each material has purported advantages in stimulating cartilage production in preclinical studies, significant differences in clinical performance are not known at this time. In contrast to the in vitro studies that evaluate cartilage growth in these hydrogels, when in combination with microfracture in the defect, these scaffolds increase the mechanical stability of the blood clot and modulate cell behaviours, such as increasing the number of cells and biologics that are trapped in the defect.

Moreover, the chemical structure of the hydrogel can influence the inhibition of fibroblasts and, ultimately, reduce scar formation in the defect while supporting cartilage development. Hydrogel chemistries that have been investigated primarily in vitro include self-assembling peptides: for example, a hydrophobic and hydrophilic alternating amino acid sequence in KLD-12 (REF. 130), TGF $\beta$ binding peptide, HSNGLPL ${ }^{131}$ and multidomain self-assembly peptides crosslinked through multivalent drugs ${ }^{130}$. Stiffer scaffold materials, including examples that replicate the overall strength of the mature tissue or, at a more detailed level, the collagen fibrillar organization, have also been engineered and tested in animal models ${ }^{108,132}$ (TABLE 1).

\section{Regenerative materials in ophthalmology}

The application of artificial materials in the eye dates back to the mid-twentieth century ${ }^{133}$. Although the consistent use of materials in the eye impedes, to a degree, the development of new biointeractive materials and regenerative strategies, it remains an active area of research. Contact lenses and intraocular lenses have a successful track record for correcting refractive error and enabling cataract replacement, respectively, using polyacrylates ${ }^{134}$ and silicone ${ }^{135,136}$. There are many examples of commercially available implants and devices used in ophthalmology that incorporate a biomaterial ${ }^{137,138}$ (TABLE 2). Despite the observed compatibility of the materials, research today is focusing on designing materials with biomimetic properties to address the challenges of contact lens discomfort and diseases such as dry eye. By mimicking or incorporating elements of the healthy ocular surface or tear film, the contact lens can be more interactive with the surrounding environment. In the lens, proliferation of capsular cells can reduce clarity and impede vision ${ }^{139}$; therefore, enhancing compatibility and guiding the surrounding lens capsule repair process using biointeractive materials would reduce potential adverse events. In the back of the eye, biomaterials have been exploited for delivery of retinal progenitor cells ${ }^{140}$ and for the drug delivery of vascular inhibitors to prevent macular degeneration ${ }^{141}$.

One of the more challenging objectives for biomaterials and tissue engineering research is engineering cornea tissue, which serves as 'the window of the eye'. Collagen is the primary component of the cornea; however, its careful organization into fibrils and orthogonal lamellae creates a transparent tissue unlike any other collagenous tissue in the body (FIG. 5). In developed countries, transplantation remains a successful clinical choice when the cornea fails, but acute, long-term chronic rejection can occur, and in most developing countries there is a severe shortage of donor tissue ${ }^{142}$. Moreover, several disease scenarios preclude cornea transplantation and repairing the tissue is the preferred route in trauma cases. The cornea has three layers: the corneal epithelium, the stroma, which comprises most of the tissue volume, and the cornea endothelium. Biomaterials in the form of membranes and hydrogels have been developed as cell culture substrates and implantation vehicles for cells to replace the epithelial layer; limbal stem cells, oral mucosa cells and corneal epithelial cells have all been tested to repair the ocular surface ${ }^{143,144}$.

For decades, purely synthetic cornea replacements were synthesized using various combinations of poly(2-hydroxyethyl methacrylate) and other acrylics

Table 1 | Selected examples of commercially available clinical products for cartilage repair

\begin{tabular}{lll}
\hline Clinical product & Company & Material treatment technology \\
\hline CartiCel & Genzyme & Collagen membranes \\
\hline ChondroGide & Geistlich & Collagen bilayer \\
\hline BioSeed-C & Biotissue & PLGA-polydioxane-fibrin \\
\hline HyloFast & Anika & Hyaluronic acid \\
\hline $\mathrm{MACl}$ & Genzyme & Collagen scaffold \\
\hline $\mathrm{Maix}$ & Matricel & Collagen-elastin \\
\hline NeoCart & Histogenix & Collagen \\
\hline Novocart 3D & Tetec & Collagen-chondroitin sulfate \\
\hline Chondron & Sewon CellOnTech & Fibrin \\
\hline CaReS & Arthro Kinetics & Collagen \\
\hline deNovo NT & Zimmer & Natural cartilage tissue \\
\hline PLGA, poly(lactic-co-glycolic acid). & &
\end{tabular}

PLGA, poly(lactic-co-glycolic acid). 
in different physical forms ${ }^{145}$, such as the 'Boston keratoprosthesis' composed of poly(methyl methacrylate) ${ }^{146}$. However, the unique immune environment of the cornea can frequently cause a melting process in which the stromal tissue dissolves and the synthetic implant is extruded from the eye. More recently, biological and biomimetic strategies are looking to provide a regenerative scaffold and wound healing option for the cornea ${ }^{147}$, such as using structural protein membranes ${ }^{148}$ and nanofibres of synthetic peptides ${ }^{149}$ (FIG. 7). Although the cornea does not have to withstand the physical forces required of articular cartilage, it has the unique requirement of clarity.

The most clinically advanced biological scaffold implant in development is a crosslinked collagen membrane. Although collagenous materials are generally opaque, these cornea mimetic collagen membranes were rendered clear by optimization of the collagen concentration and crosslinking conditions. These scaffolds have been used to make in vitro cornea tissues and have been implanted in an acellular form in large animals ${ }^{150-152}$. This scaffold was then translated to clinical testing, in which it remained stably integrated with native tissue and restored tear film, nerve regeneration, and touch sensitivity and vision in six patients over 24 months post-surgery ${ }^{153}$. However, the collagen in these implants has a lower density and is not organized into fibrillar lamellae as it is in the native tissue, and, as a result, the implants do not have the mechanical strength necessary for direct suturing, which led to practical challenges in clinical testing. Vitrification - water evaporation at a controlled temperature and humidity - applied to collagen hydrogels can increase the fibrillar organization to enhance mechanical properties and promote cornea stromal keratocyte phenotype maintenance ${ }^{154}$. Materials development for the cornea continues with the goal of creating a transparent implant that has adequate mechanical strength for implantation. Copolymers of collagen and proteoglycan additives are being investigated in combination with the collagen scaffolds to mimic the native tissue more closely. In addition, the organization of collagen using its biophysical properties and molecular crowding can be used to create thin collagen membranes with lamellae similar to those of the native cornea, albeit much thinner than the native tissue ${ }^{155}$.

Other materials that are being considered for cornea scaffolds include silk and the traditional degradable polyesters ${ }^{156}$, some of which are being organized into orthogonal sheets to mimic the native tissue. Tissuederived materials, which were described earlier, are in clinical use today in the field of ophthalmology, including amniotic membrane and acellular human- and animal-derived cornea tissue ${ }^{157,158}$, and conjunctiva tissue $^{159}$. In contrast to synthetic biomaterials that rebuild the native structure from a 'bottom-up' perspective, tissue-derived materials take a 'top-down' approach to benefit from the existing complex, the native tissue structures and the biological benefits of the ECM. The processing of full-thickness cornea tissue through decellularization is also being explored as an option to produce the implant strength and matrix organization complexity needed for proper cornea function ${ }^{160,161}$.

\section{Future perspectives}

Discoveries in cell and developmental biology that define key immune mediators of repair and regeneration can be considered in the design of biomaterial scaffolds that modulate the immune response: a concept emerging as biomaterials-directed regenerative immunology. Materials that target the immune system have, to date,

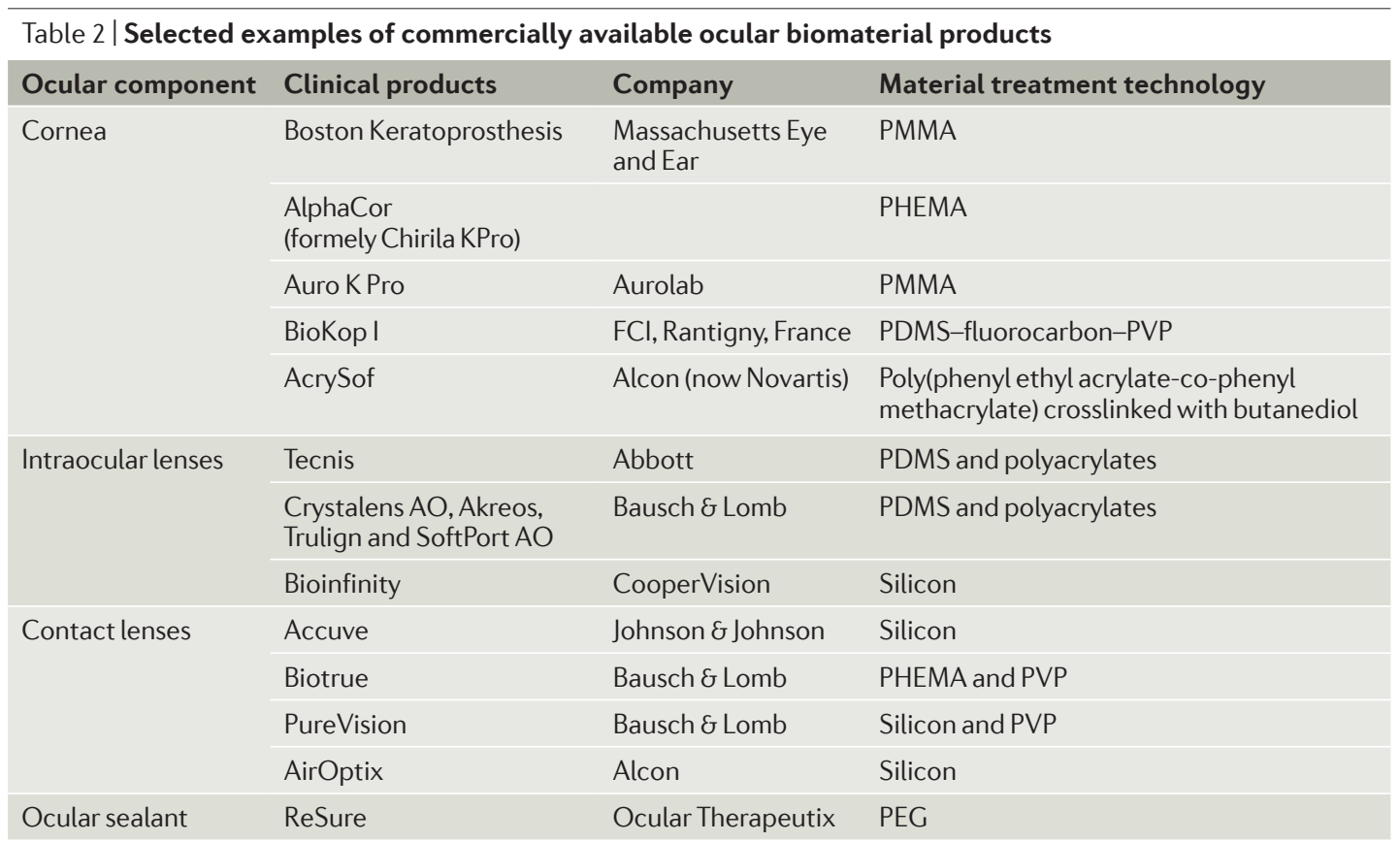

PDMS, poly(dimethylsiloxane); PEG, poly(ethylene glycol); PHEMA, poly(2-hydroxyethyl methacrylate); PMMA, poly(methyl methacrylate); PVP, poly(vinyl pyrrolidone). 
a Epithelial surface of the cornea

Synthetic polymers as culture materials
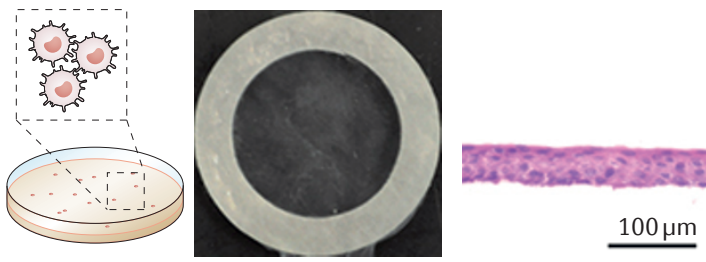

$100 \mu \mathrm{m}$

Collagen membrane with organized fibrillar structure
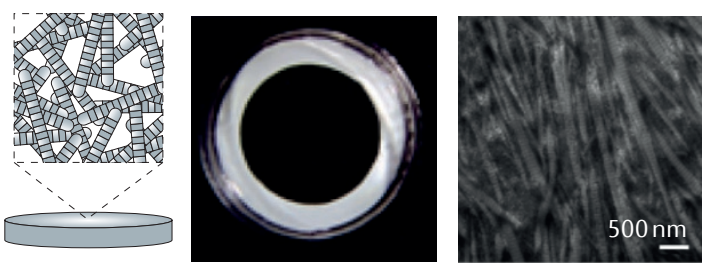

Biological gel to deliver cells
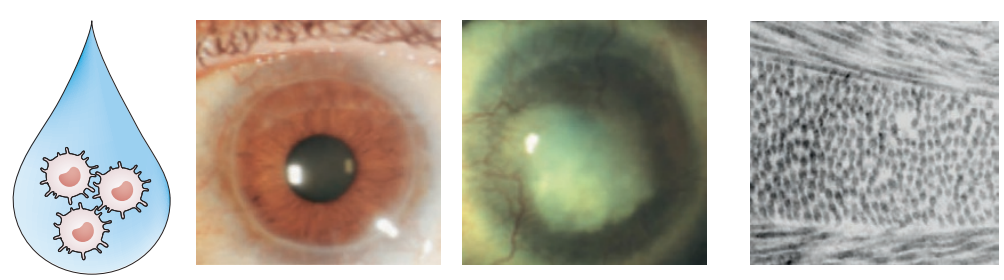

Synthetic hydrogel
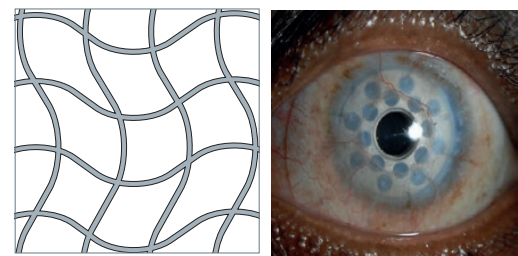

Biological hydrogel

Organized collagen layers
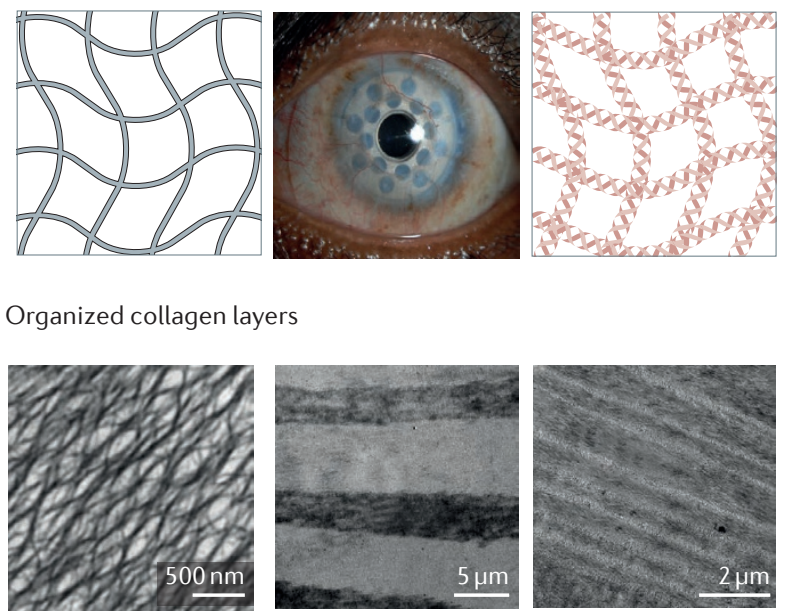

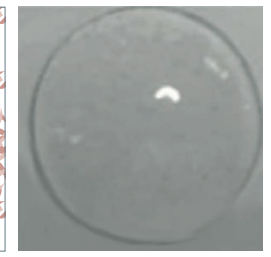

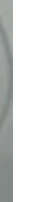

Figure 7 | Corneal tissue engineering. a | Biomaterial membranes are a vehicle to culture and deliver cells for rebuilding the surface of the cornea. For example, epithelial cell sheets are created on synthetic materials ${ }^{192}$; fibrin glue supports the culture and delivery of limbal epithelial stem cells to the surface of the cornea ${ }^{144}$; and organized collagen membranes produced through vitrification (a controlled drying process) of collagen gels contain nanostructured collagen that supports a differentiated cell phenotype ${ }^{154,193} \cdot \mathbf{b}$ | Bulk stromal replacement and tissue engineering is performed with both synthetic ${ }^{194}$ and biological ${ }^{153}$ hydrogels. These synthetic hydrogels are made of polymers such as poly(methyl methacrylate) $)^{135}$, whereas the biological hydrogels are largely derived from the manipulation of collagen. Collagen-based hydrogels can be synthesized from fibrillar collagen via chemical crosslinking ${ }^{151}$, molecular crowding ${ }^{155}$, vitrification ${ }^{154}$ or by the processing of a full native cornea by decellularization ${ }^{160,161}$. Transmission electron micrographs of the control (third row; left image) and a full-thicknessprocessed cornea following decellurization (middle image) are shown. A porcine cornea after decellularization and dehydration with sucrose is shown (right image). Part a (first row) is from REF. 192, Nature Publishing Group. Part a (second row) is reprinted with permission from REF. 144, Massachusetts Medical Society. Part a (third row; middle panel) is reproduced with permission from REF. 154; permission conveyed through Copyright Clearance Center, Inc. Part a (third row; right panel) is reproduced with permission from REF. 193, Wiley. Part b (first row; synthetic hydrogel) is courtesy of J. Chodosh, Harvard Medical School, USA. Part b (first row; biological hydrogel) is adapted with permission from REF. 153, AAAS. Part b (second row) is reproduced with permission from REF. 155, Elsevier. Part b (third row; left and middle panels) is reproduced with permission from REF. 161; the publisher for this copyrighted material is Mary Ann Liebert, Inc. Part b (third row, right panel) is reproduced with permission from REF. 160, Public Library of Science. publishers.

been primarily in the form of nano- and microparticles for applications in cancer immunology and vaccines. For example, nanoparticles are targeting and stimulating $\mathrm{CD}^{+}$cytotoxic $\mathrm{T}$ lymphocytes to enhance tumour and cancer cell clearance ${ }^{162,163}$. Polymeric nanoparticles are engineered to contain both the antigen of interest as well as co-stimulatory molecules to fully activate an immune response. These particles are ingested and processed by antigen-presenting cells (APCs), such as dendritic cells and macrophages, and then APCs present the antigen on major histocompatibility complexes to cognate $\mathrm{T}$ cell receptors. These targeting strategies are also being applied to vaccine development and tolerogenic treatments for autoimmune disease ${ }^{164-166}$. Materials are used to engineer completely synthetic APCs ${ }^{167,168}$, and through careful analysis, the optimal shape and size for efficient $\mathrm{T}$ cell activation was determined.

On a larger scale, polymer implants are also working to educate the immune system in a similar manner ${ }^{169,170}$. Macroscale therapeutic biomaterial implants are currently in clinical testing ${ }^{171}$. These subcutaneously implanted biodegradable wafers are formulated to contain tumour lysate as a source of tumour antigen together with granulocyte-macrophage colony-stimulating factor 


\section{Box 2 | Key points}

- Biomaterial scaffolds are designed to guide cell behaviour and thus promote tissue repair for regenerative medicine applications

- New strategies for the chemical and physical modification of scaffolds have enabled fundamental studies on cellular responses to biomaterials and in vitro tissue model systems

- Clinical translation of regenerative biomaterials has been limited, but clinical examples provide key insights into therapeutic mechanisms of action and future design parameters

- The immune system may be a key regulator of not only biocompatibility of materials but also biomaterials-mediated tissue regeneration
Many advances in engineering biomaterials for regenerative medicine have been seen in recent decades (BOX 2). Fundamental properties of cell responses to biochemical and biophysical cues have been delineated with careful structure-function studies. Sophisticated techniques now allow replication of the structural organization of complex, native tissue in biomaterials and also the mimicking of developmental biology cues that are embedded in the biomaterial scaffold. However, we must not neglect the role of biology and 'overengineer' materials such that they actually impede the innate processes of cells and tissues that still know how to rebuild tissues better than we do. Furthermore, the manipulation and combination of polymers to create new scaffolds generally provide incremental improvements in matrix production in vitro that may not even translate to preclinical or clinical improvements. The fields of biomaterials and regenerative medicine also have the potential to mature beyond the trial-and-error design method by integrating big data strategies for materials design, cell and tissue responses, and, most importantly, to incorporate lessons from translation. Thus, smart materials may not always be more complex or sophisticated, but their design takes into consideration structure-function relationships, successes and failures in translation, and the primary factors contributing to efficacy.
1. Thurston, A. J. Pare and prosthetics: the early history of artificial limbs. ANZ J. Surg. 77, 1114-1119 (2007).

2. Mazzola, R. F. \& Marcus, S. History of total nasal reconstruction with particular emphasis on the folded forehead flap technique. Plast. Reconstr. Surg. 72 408-414 (1983)

3. Langer, R. \& Vacanti, J. P. Tissue engineering. Science 260, 920-926 (1993)

4. Langer, R. \& Tirrell, D. A. Designing materials for biology and medicine. Nature 428, 487-492 (2004).

5. Mikos, A. G. et al. Engineering complex tissues. Tissue Eng. 12, 3307-3339 (2006).

6. Pashuck, E. T. \& Stevens, M. M. Designing regenerative biomaterial therapies for the clinic. Sci. Transl. Med. 4, 160sr4 (2012)

7. Macchiarini, P. et al. Clinical transplantation of a tissue engineered airway. Lancet 372, 2023-2030 (2008).

8. Warnke, P. H. et al. Growth and transplantation of a custom vascularised bone graft in a man. Lancet 364 766-770 (2004)

9. Haraguchi, Y. et al. Fabrication of functional threedimensional tissues by stacking cell sheets in vitro. Nat. Protoc. 7, 850-858 (2012).

10. Fennema, E., Rivron, N., Rouwkema, J., van Blitterswijk, C. \& de Boer, J. Spheroid culture as a too for creating 3D complex tissues. Trends Biotechnol. 31, 108-115 (2013)

11. Liu, J. S. \& Gartner, Z. J. Directing the assembly of spatially organized multicomponent tissues from the bottom up. Trends Cell Biol. 22, 683-691 (2012).

12. Stevens, M. M. et al. In vivo engineering of organs: the bone bioreactor. Proc. Natl Acad. Sci. USA 102, 11450-11455 (2005).

13. Lutolf, M. P. \& Hubbell, J. A. Synthetic biomaterials as instructive extracellular microenvironments for morphogenesis in tissue engineering. Nat. Biotechnol. 23, 47-55 (2005).

14. Pittenger, M. F. et al. Multilineage potential of adult human mesenchymal stem cells. Science $\mathbf{2 8 4}$ 143-147 (1999).

15. Ma, Z. et al. Self-organizing human cardiac microchambers mediated by geometric confinement. Nat. Commun. 6, 7413 (2015)

16. Yang, C., Tibbitt, M. W., Basta, L. \& Anseth, K. S Mechanical memory and dosing influence stem cell fate. Nat. Mater. 13, 645-652 (2014).
17. Benoit, D. S., Schwartz, M. P., Durney, A. R. \& Anseth, K. S. Small functional groups for controlled differentiation of hydrogel-encapsulated human mesenchymal stem cells. Nat. Mater. 7, 816-823 (2008)

18. Singh, A., Zhan, J. A., Ye, Z. Y. \& Elisseeff, J. H. Modular multifunctional poly(ethylene glycol) hydrogels for stem cell differentiation. Adv. Funct. Mater. 23, 575-582 (2013).

19. Kilian, K. A. \& Mrksich, M. Directing stem cell fate by controlling the affinity and density of ligand-receptor interactions at the biomaterials interface. Angew. Chem. Int. Ed. Engl. 51, 4891-4895 (2012).

20. Schense, J. C., Bloch, J., Aebischer, P. \& Hubbell, J. A. Enzymatic incorporation of bioactive peptides into fibrin matrices enhances neurite extension. Nat. Biotechnol. 18, 415-419 (2000).

21. Hwang, N. S. et al. In vivo commitment and functional tissue regeneration using human embryonic stem cellderived mesenchymal cells. Proc. Natl Acad. Sci. USA 105, 20641-20646 (2008).

22. Lutolf, M. P., Gilbert, P. M. \& Blau, H. M. Designing materials to direct stem-cell fate. Nature $\mathbf{4 6 2}$ 433-441 (2009).

23. MacDonald, B. T., Tamai, K. \& He, X. Wnt/B-catenin signaling: components, mechanisms, and diseases. Dev. Cell 17, 9-26 (2009).

24. de Boer, J. et al. Wnt signaling inhibits osteogenic differentiation of human mesenchymal stem cells. Bone 34, 818-826 (2004).

25. Azarin, S. M. et al. Modulation of Wnt/ $\beta$-catenin signaling in human embryonic stem cells using a 3D microwell array. Biomaterials 33, 2041-2049 (2012).

26. Flaim, C. J., Chien, S. \& Bhatia, S. N. An extracellular matrix microarray for probing cellular differentiation. Nat. Methods 2, 119-125 (2005).

27. Gobaa, S. et al. Artificial niche microarrays for probing single stem cell fate in high throughput. Nat. Methods 8, 949-955 (2011)

28. Somoza, R. A., Welter, J. F., Correa, D. \& Caplan, A. I. Chondrogenic differentiation of mesenchymal stem cells: challenges and unfulfilled expectations. Tissue Eng. Part B Rev. 20, 596-608 (2014)

29. Caplan, A. I. Adult mesenchymal stem cells for tissue engineering versus regenerative medicine. J. Cell. Physiol. 213, 341-347 (2007)
30. Meirelles Lda S., Fontes, A. M. Covas, D. T \& Caplan, A. I. Mechanisms involved in the therapeutic properties of mesenchymal stem cells. Cytokine Growth Factor Rev. 20, 419-427 (2009).

31. Aggarwal, S. \& Pittenger, M. F. Human mesenchymal stem cells modulate allogeneic immune cell responses. Blood 105, 1815-1822 (2005).

32. Schmalzried, T. P. \& Callaghan, J. J. Wear in total hip and knee replacements. J. Bone Joint Surg. Am. 81 115-136 (1999)

33. Anderson, J. M. \& Miller, K. M. Biomaterial biocompatibility and the macrophage. Biomaterials 5 5-10 (1984)

34. Anderson, J. M. Inflammatory response to implants. ASAIO Trans. 34, 101-107 (1988).

35. Marchant, R. et al. In vivo biocompatibility studies. I. The cage implant system and a biodegradable hydrogel. J. Biomed. Mater. Res. 17, 301-325 (1983)

36. Marchant, R. E., Anderson, J. M., Phua, K. \& Hiltner, A. In vivo biocompatibility studies. II. Biomer: preliminary cell adhesion and surface characterization studies. J. Biomed. Mater. Res. 18, 309-315 (1984)

37. Marchant, R. E., Miller, K. M \& Anderson, J. M. In vivo biocompatibility studies. V. In vivo leukocyte interactions with Biomer. J. Biomed. Mater. Res. 18 1169-1190 (1984)

38. Marchant, R. E., Anderson, J. M. \& Dillingham, E. O. In vivo biocompatibility studies. VII. Inflammatory response to polyethylene and to a cytotoxic polyvinylchloride. J. Biomed. Mater. Res. 20, 37-50 (1986).

39. Bauer, T. W., Geesink, R., Zimmerman, R. \& McMahon, J. T. Hydroxyapatite-coated femoral stems. Histological analysis of components retrieved at autopsy. J. Bone Joint Surg. Am. 73, 1439-1452 (1991).

40. Reid, B. et al. PEG hydrogel degradation and the role of the surrounding tissue environment. J. Tissue Eng. Regen. Med. 9, 315-318 (2013).

41. Hillel, A. T. et al. Photoactivated composite biomaterial for soft tissue restoration in rodents and in humans. Sci. Transl. Med. 3, 93 ra67 (2011).

42. Gosselin, D. et al. Environment drives selection and function of enhancers controlling tissue-specific macrophage identities. Cell 159, 1327-1340 (2014) 
43. Lavin, Y. et al. Tissue-resident macrophage enhancer landscapes are shaped by the local microenvironment. Cell 159, 1312-1326 (2014)

44. Beachley, V. Z. et al. Tissue matrix arrays for highthroughput screening and systems analysis of cell function. Nat. Methods 12, 1197-1204 (2015).

45. LaBarge, M. A. et al. Human mammary progenitor cell fate decisions are products of interactions with combinatorial microenvironments. Integr. Biol. (Camb.) 1, 70-79 (2009)

46. Seliktar, D. Designing cell-compatible hydrogels for biomedical applications. Science 336, 1124-1128 (2012).

47. Saraf, A. et al. Fabrication of nonwoven coaxial fiber meshes by electrospinning. Tissue Eng. Part C Methods 15, 333-344 (2009).

48. Moutos, F. T., Freed, L. E. \& Guilak, F. A biomimetic three-dimensional woven composite scaffold for functional tissue engineering of cartilage. Nat. Mater. 6, 162-167 (2007).

49. Rnjak-Kovacina, J. et al. Lyophilized silk sponges: a versatile biomaterial platform for soft tissue engineering ACS Biomater. Sci. Eng. 1, 260-270 (2015).

50. Dahlin, R. L. et al. Articular chondrocytes and mesenchymal stem cells seeded on biodegradable scaffolds for the repair of cartilage in a rat osteochondral defect model. Biomaterials 35, 7460-7469 (2014).

51. Engler, A. J., Sen, S., Sweeney, H. L. \& Discher, D. E. Matrix elasticity directs stem cell lineage specification. Cell 126, 677-689 (2006).

52. Beck, J. N., Singh, A., Rothenberg, A. R. Elisseeff, J. H. \& Ewald, A. J. The independent roles of mechanical, structural and adhesion characteristics of 3D hydrogels on the regulation of cancer invasion and dissemination. Biomaterials 34, 9486-9495 (2013).

53. Levorson, E. J., Santoro, M., Kasper, F. K. \& Mikos, A. G. Direct and indirect co-culture of chondrocytes and mesenchymal stem cells for the generation of polymer/extracellular matrix hybrid constructs. Acta Biomater. 10, 1824-1835 (2014)

54. Purcell, B. P. et al. Injectable and bioresponsive hydrogels for on-demand matrix metalloproteinase inhibition. Nat. Mater. 13, 653-661 (2014)

55. Khetan, S. et al. Degradation-mediated cellular traction directs stem cell fate in covalently crosslinked threedimensional hydrogels. Nat. Mater. 12, 458-465 (2013).

56. Rape, A. D., Zibinsky, M., Murthy, N. \& Kumar, S. A synthetic hydrogel for the high-throughput study of cell-ECM interactions. Nat. Commun. 6, 8129 (2015).

57. Pace, L. A., Plate, J. F., Smith, T. L. \& Van Dyke, M. E. The effect of human hair keratin hydrogel on early cellular response to sciatic nerve injury in a rat model. Biomaterials 34, 5907-5914 (2013).

58. Wang, S. et al. A new nerve guide conduit material composed of a biodegradable poly(phosphoester). Biomaterials 22, 1157-1169 (2001).

59. Altman, G. H. et al. Silk matrix for tissue engineered anterior cruciate ligaments. Biomaterials 23 4131-4141 (2002)

60. Vunjak-Novakovic, G., Altman, G., Horan, R. \& Kaplan, D. L. Tissue engineering of ligaments. Annu. Rev. Biomed. Eng. 6, 131-156 (2004).

61. Murphy, S. V. \& Atala, A. 3D bioprinting of tissues and organs. Nat. Biotechnol. 32, 773-785 (2014).

62. Lee, C. H. et al. Protein-releasing polymeric scaffolds induce fibrochondrocytic differentiation of endogenous cells for knee meniscus regeneration in sheep. Sci. Transl. Med. 6, 266ra171 (2014).

63. Reiffel, A. J. et al. High-fidelity tissue engineering of patient-specific auricles for reconstruction of pediatric microtia and other auricular deformities. PLOS ONE 8 , e56506 (2013).

64. DeForest, C. A. \& Anseth, K. S. Cytocompatible clickbased hydrogels with dynamically tunable properties through orthogonal photoconjugation and photocleavage reactions. Nat. Chem. 3, 925-931 (2011).

65. Baskin, J. M. et al. Copper-free click chemistry for dynamic in vivo imaging. Proc. Natl Acad. Sci. USA 104, 16793-16797 (2007)

66. Li, W. \& Ding, S. Small molecules that modulate embryonic stem cell fate and somatic cell reprogramming. Trends Pharmacol. Sci. 31, 36-45 (2010).

67. Chen, S., Zhang, Q., Wu, X., Schultz, P. G. \& Ding, S Dedifferentiation of lineage-committed cells by a small molecule. J. Am. Chem. Soc. 126, 410-411 (2004).

68. Li, K. et al. Small molecules facilitate the reprogramming of mouse fibroblasts into pancreatic lineages. Cell Stem Cell 14, 228-236 (2014).

69. Gilbert, T. W., Sellaro, T. L. \& Badylak, S. F. Decellularization of tissues and organs. Biomaterials 27, 3675-3683 (2006).
70. Badylak, S. F., Freytes, D. O. \& Gilbert, T. W. Extracellular matrix as a biological scaffold material: structure and function. Acta Biomater. 5, 1-13 (2009).

71. Wolf, M. T., Dearth, C. L., Sonnenberg, S. B., Loboa, E. G. \& Badylak, S. F. Naturally derived and synthetic scaffolds for skeletal muscle reconstruction Adv. Drug Deliv. Rev. 84, 208-221 (2015)

72. Naba, A. et al. The matrisome: in silico definition and in vivo characterization by proteomics of normal and tumor extracellular matrices. Mol. Cell Proteomics 11 M111.014647 (2012).

73. Ahn, J. M. et al. Proteogenomic analysis of human chromosome 9-encoded genes from human samples and lung cancer tissues. J. Proteome Res. 13 , 137-146 (2014).

74. Murthy, K. R. et al. Proteomic analysis of human vitreous humor. Clin. Proteom. 11, 29 (2014).

75. Miller, J. S. et al. Rapid casting of patterned vascular networks for perfusable engineered three-dimensional tissues. Nat. Mater. 11, 768-774 (2012).

76. Liu, Q. et al. Porous nanofibrous poly(L-lactic acid) scaffolds supporting cardiovascular progenitor cells for cardiac tissue engineering. Acta Biomater. 26 205-114 (2015).

77. Anderson, J. M. Biological responses to materials. Annu. Rev. Mater. Res. 31, 81-110 (2001).

78. Anderson, J. M., Rodriguez, A. \& Chang, D. T. Foreign body reaction to biomaterials. Semin. Immunol. 20 , 86-100 (2008).

79. Gordon, S. Alternative activation of macrophages. Nat. Rev. Immunol. 3, 23-35 (2003).

80. Martinez, F. O., Gordon, S., Locati, M. \& Mantovani, A. Transcriptional profiling of the human monocyte-to-macrophage differentiation and polarization: new molecules and patterns of gene expression. J. Immunol. 177, 7303-7311 (2006).

81. Bryers, J. D., Giachelli, C. M. \& Ratner, B. D. Engineering biomaterials to integrate and heal: the biocompatibility paradigm shifts. Biotechnol. Bioeng. 109, 1898-1911 (2012)

82. Brown, B. N., Ratner, B. D., Goodman, S. B., Amar, S. \& Badylak, S. F. Macrophage polarization: an opportunity for improved outcomes in biomaterials and regenerative medicine. Biomaterials 33, 3792-3802 (2012)

83. Murray, P. J. et al. Macrophage activation and polarization: nomenclature and experimental guidelines. Immunity 41, 14-20 (2014).

84. Otis, J. S. et al. Pro-inflammatory mediation of myoblast proliferation. PLOS ONE 9, e92363 (2014).

85. Tidball, J. G. \& Villalta, S. A. Regulatory interactions between muscle and the immune system during muscle regeneration. Am. J. Physiol. Regul. Integr Comp. Physiol. 298, R1173-1187 (2010).

86. Matzinger, P. Friendly and dangerous signals: is the tissue in control? Nat. Immunol. 8, 11-13 (2007).

87. Matzinger, P. \& Kamala, T. Tissue-based class control: the other side of tolerance. Nat. Rev. Immunol. 11 221-230 (2011).

88. Ma, J. et al. Regulation of macrophage activation. Cell. Mol. Life Sci. 60, 2334-2346 (2003).

89. Rengarajan, J., Szabo, S. J. \& Glimcher, L. H. Transcriptional regulation of Th $1 /$ Th2 polarization. Immunol. Today 21, 479-483 (2000).

90. Godwin, J. W., Pinto, A. R. \& Rosenthal, N. A. Macrophages are required for adult salamander limb regeneration. Proc. Natl Acad. Sci. USA 110 , 9415-9420 (2013).

91. Heredia, J. E. et al. Type 2 innate signals stimulate fibro/adipogenic progenitors to facilitate muscle regeneration. Cell 153, 376-388 (2013).

92. Sadtler, K. et al. Developing a pro-regenerative biomaterial scaffold microenvironment requires T helper 2 cells. Science 352, 366-370 (2016)

93. McNally, A. K. \& Anderson, J. M. Interleukin- 4 induces foreign body giant cells from human monocytes/ macrophages. Differential lymphokine regulation of macrophage fusion leads to morphological variants of multinucleated giant cells. Am. J. Pathol. 147 1487-1499 (1995)

94. Epelman, S., Liu, P. P. \& Mann, D. L. Role of innate and adaptive immune mechanisms in cardiac injury and repair. Nat. Rev. Immunol. 15, 117-129 (2015).

95. Schlundt, C. et al. Macrophages in bone fracture healing: their essential role in endochondral ossification. Bone http://dx.doi.org/10.1016/j. bone.2015.10.019 (2015)

96. Konnecke, I. et al. T and B cells participate in bone repair by infiltrating the fracture callus in a two-wave fashion. Bone 64, 155-165 (2014).
97. Reinke, S. et al. Terminally differentiated $C D 8+T$ cells negatively affect bone regeneration in humans. Sci. Transl. Med. 5, 177 ra36 (2013).

98. Gaudilliere, B. et al. Clinical recovery from surgery correlates with single-cell immune signatures. $\mathrm{SCl}$. Transl. Med. 6, 255ra131 (2014)

99. Guilak, F. et al. Control of stem cell fate by physical interactions with the extracellular matrix. Cell Stem Cell 5, 17-26 (2009).

100. Khademhosseini, A. \& Langer, R. Microengineered hydrogels for tissue engineering. Biomaterials $\mathbf{2 8}$ 5087-5092 (2007).

101. Elliott, M. J. et al. Stem-cell-based, tissue engineered tracheal replacement in a child: a 2-year follow-up study. Lancet 380, 994-1000 (2012).

102. Morrison, R. J. et al. Mitigation of tracheobronchomalacia with 3D-printed personalized medical devices in pediatric patients. Sci. Transl. Med. 7, 285 ra64 (2015)

103. Gosset, M., Berenbaum, F., Thirion, S. \& Jacques, C. Primary culture and phenotyping of murine chondrocytes. Nat. Protoc. 3, 1253-1260 (2008).

104. Bednarz, J., Weich, H. A., Rodokanaki-von Schrenck, A. \& Engelmann, K. Expression of genes coding growth factors and growth factor receptors in differentiated and dedifferentiated human corneal endothelial cells. Cornea 14, 372-381 (1995).

105. Wong, B. L. et al. Biomechanics of cartilage articulation effects of lubrication and degeneration on shear deformation. Arthritis Rheum. 58, 2065-2074 (2008).

106. Seror, J., Zhu, L., Goldberg, R., Day, A. J. \& Klein, J. Supramolecular synergy in the boundary lubrication of synovial joints. Nat. Commun. 6, 6497 (2015).

107. Xie, X. et al. Comparative evaluation of MSCs from bone marrow and adipose tissue seeded in PRPderived scaffold for cartilage regeneration. Biomaterials 33, 7008-7018 (2012)

108. Jeng, L., Hsu, H.-P. \& Spector, M. Tissue-engineered cartilaginous constructs for the treatment of caprine cartilage defects, including distribution of laminin and type IV collagen. Tissue Eng. Part A 19, 2267-2274 (2013).

109. Kon, E. et al. Osteochondral regeneration with a nove aragonite-hyaluronate biphasic scaffold: up to 12 -month follow-up study in a goat model. J. Orthop. Surg. Res. 10, 81 (2015).

110. Navarro, M., Michiardi, A., Castano, O. \& Planell, J. A. Biomaterials in orthopaedics. J. R. Soc. Interface 5 , 1137-1158 (2008)

111. Brittberg, M. et al. Treatment of deep cartilage defects in the knee with autologous chondrocyte transplantation. N. Engl. J. Med. 331, 889-895 (1994).

112. Peterson, L., Minas, T., Brittberg, M. \& Lindahl, A Treatment of osteochondritis dissecans of the knee with autologous chondrocyte transplantation: results at two to ten years. J. Bone Joint Surg. Am. 85-A (Suppl. 2), 17-24 (2003).

113. Cole, B. J., Pascual-Garrido, C. \& Grumet, R. C. Surgical management of articular cartilage defects in the knee. Instr. Course Lect. 59, 181-204 (2010).

114. Dewan, A. K., Gibson, M. A., Elisseeff, J. H. \& Trice, A. E. Evolution of autologous chondrocyte repair and comparison to other cartilage repair techniques. Biomed. Res. Int. (2014).

115. Lee, H.-i., Pietrasik, J., Sheiko, S. S. \& Matyjaszewski, K. Stimuli-responsive molecular brushes. Prog. Polym. Sci. 35, 24-44 (2010).

116. Banquy, X., Burdyn`ska, J., Lee, D. W. Matyjaszewski, K. \& Israelachvili, J. Bioinspired bottlebrush polymer exhibits low friction and Amontons-like behavior. J. Am. Chem. Soc. 136, 6199-6202 (2014)

117. Jahn, S. \& Klein, J. Hydration lubrication: the macromolecular domain. Macromolecules 48 , 5059-5075 (2015).

118. Singh, A. et al. Enhanced lubrication on tissue and biomaterial surfaces through peptide-mediated binding of hyaluronic acid. Nat. Mater. 13, 988-995 (2014).

119. Liu, X., Jin, X. \& Ma, P. X. Nanofibrous hollow microspheres self-assembled from star-shaped polymers as injectable cell carriers for knee repair. Nat. Mater. 10, 398-406 (2011)

120. Crawford, D. C., DeBerardino, T. M. \& 3rd Williams, R. J. NeoCart, an autologous cartilage tissue implant, compared with microfracture for treatment of distal femoral cartilage lesions: an FDA phase-II prospective, randomized clinical trial after two years. J. Bone Joint Surg. Am. 94, 979-989 (2012).

121. Coburn, J. M., Gibson, M., Monagle, S., Patterson, Z. \& Elisseeff, J. H. Bioinspired nanofibers support chondrogenesis for articular cartilage repair. Proc. Natl Acad. Sci. USA 109, 10012-10017 (2012). 
122. Foldager, C. B., Gomoll, A. H., Lind, M. \& Spector, M. Cell seeding densities in autologous chondrocyte implantation techniques for cartilage repair. Cartilage 3, 108-117 (2012)

123. Obradovic, B. et al. Integration of engineered cartilage. J. Orthop. Res. 19, 1089-1097 (2001).

124. Ladet, S., David, L. \& Domard, A. Multi-membrane hydrogels. Nature 452, 76-79 (2008).

125. Strehin, I., Nahas, Z., Arora, K., Nguyen, T. \& Elisseeff, J. A versatile $\mathrm{pH}$ sensitive chondroitin sulfate-PEG tissue adhesive and hydrogel. Biomaterials 31, 2788-2797 (2010).

126. Sharma, B. et al. Human cartilage repair with a photoreactive adhesive-hydrogel composite. Sci. Transl. Med. 5, 167ra6 (2013).

127. Wang, D. A. et al. Multifunctional chondroitin sulphate for cartilage tissue-biomaterial integration. Nat. Mater. 6, 385-392 (2007)

128. Chenite, A. et al. Novel injectable neutral solutions of chitosan form biodegradable gels in situ. Biomaterials 21, 2155-2161 (2000).

129. Almany, L. \& Seliktar, D. Biosynthetic hydrogel scaffolds made from fibrinogen and polyethylene glycol for 3D cell cultures. Biomaterials 26 , 2467-2477 (2005).

130. Kumar, V. A. et al. Drug-triggered and cross-linked self-assembling nanofibrous hydrogels. J. Am. Chem. Soc. 137, 4823-4830 (2015).

131. Shah, R. N. et al. Supramolecular design of selfassembling nanofibers for cartilage regeneration. Proc. Natl Acad. Sci. USA 107, 3293-3298 (2010).

132. Jones, A. R. et al. Modulation of lubricin biosynthesis and tissue surface properties following cartilage mechanical injury. Arthritis Rheum. 60, 133-142 (2009).

133. Lowther, G. E. Contact Lenses: Procedures and Techniques (Butterworth-Heinemann, 1992).

134. Ridley, H. Intra-ocular acrylic lenses; a recent development in the surgery of cataract. $\mathrm{Br}$. J. Ophthalmol. 36, 113-122 (1952).

135. Chehade, M. \& Elder, M. J. Intraocular lens materials and styles: a review. Aust. N. Z. J. Ophthalmol. 25 255-263 (1997).

136. Milauskas, A. T. Silicone intraocular-lens implant discoloration in humans. Arch. Ophthalmol. 109, 913-913 (1991).

137. Lloyd, A. W., Faragher, R. G. \& Denyer, S. P. Ocular biomaterials and implants. Biomaterials 22, 769-785 (2001).

138. Lace, R., Murray-Dunning, C. \& Williams, R. Biomaterials for ocular reconstruction. J. Mater. Sci. 50, 1523-1534 (2015).

139. Apple, D. J. et al. Posterior capsule opacification. Surv. Ophthalmol. 37, 73-116 (1992).

140. Ballios, B. G., Cooke, M. J., van der Kooy, D. \& Shoichet, M. S. A hydrogel-based stem cell delivery system to treat retinal degenerative diseases. Biomaterials 31, 2555-2564 (2010).

141. Aiello, L. P. et al. Suppression of retina neovascularization in vivo by inhibition of vascular endothelial growth factor (VEGF) using soluble VEGFreceptor chimeric proteins. Proc. Natl Acad. Sci. USA 92, 10457-10461 (1995)

142. Tan, D. T. H., Dart, J. K. G., Holland, E. J. \& Kinoshita, S. Corneal transplantation. Lancet 379 1749-1761 (2012)

143. Nishida, K. et al. Corneal reconstruction with tissueengineered cell sheets composed of autologous oral mucosal epithelium. N. Engl. J. Med. 351, 1187-1196 (2004).

144. Rama, P. et al. Limbal stem-cell therapy and longterm corneal regeneration. N. Engl. J. Med. 363, 147-155 (2010)

145. Chirila, T. V. et al. Poly(2-hydroxyethyl methacrylate) sponges as implant materials: in vivo and in vitro evaluation of cellular invasion. Biomaterials $\mathbf{1 4}$, 26-38 (1993)

146. Yaghouti, F. et al. Keratoprosthesis: preoperative prognostic categories. Cornea 20, 19-23 (2001).

147. Elisseeff, J., Madrid, M. G., Lu, Q., Chae, J. J. \& Guo, Q. Future perspectives for regenerative medicine in ophthalmology. Middle East Afr. J. Ophthalmol. 20, 38-45 (2013).

148. Borrelli, M. et al. Keratin films for ocular surface reconstruction: evaluation of biocompatibility in an in vivo model. Biomaterials 42, 112-120 (2015).

149. Uzunalli, G. et al. Bioactive self-assembled peptide nanofibers for corneal stroma regeneration. Acta Biomater. 10, 1156-1166 (2014).

150. Chen, W. et al. Comparison of fresh corneal tissue versus glycerin-cryopreserved corneal tissue in deep anterior lamellar keratoplasty. Invest. Ophthalmol. Vis. Sci. 51, 775-781 (2010)

151. Merrett, K. et al. Tissue-engineered recombinant human collagen-based corneal substitutes for implantation: performance of type I versus type III collagen. Invest. Ophthalmol. Vis. Sci. 49 , 3887-3894 (2008)

152. Luo, H. L. et al. Construction of tissue-engineered cornea composed of amniotic epithelial cells and acellular porcine cornea for treating corneal alkali burn. Biomaterials 34, 6748-6759 (2013).

153. Fagerholm, P. et al. A biosynthetic alternative to human donor tissue for inducing corneal regeneration: 24-month follow-up of a phase 1 clinical study. Sci. Transl. Med. 2, 46ra61 (2010).

154. Takezawa, T., Ozaki, K., Nitani, A., Takabayashi, C. \& Shimo-Oka, T. Collagen vitrigel: a novel scaffold that can facilitate a three-dimensional culture for reconstructing organoids. Cell Transplant 13 463-473 (2004).

155. Saeidi, N. et al. Molecular crowding of collagen: a pathway to produce highly-organized collagenous structures. Biomaterials 33, 7366-7374 (2012)

156. Lawrence, B. D., Marchant, J. K., Pindrus, M. A., Omenetto, F. G. \& Kaplan, D. L. Silk film biomaterials for cornea tissue engineering. Biomaterials 30 1299-1308 (2009)

157. Fu, Y., Fan, X., Chen, P., Shao, C. \& Lu, W. Reconstruction of a tissue-engineered cornea with porcine corneal acellular matrix as the scaffold. Cells Tissues Organs 191, 193-202 (2010).

158. Koizumi, N. et al. Cultivation of corneal epithelial cells on intact and denuded human amniotic membrane. Invest. Ophthalmol. Vis. Sci. 41 2506-2513 (2000).

159. Zhao, H., Qu, M., Wang, Y., Wang, Z. \& Shi, W. Xenogeneic acellular conjunctiva matrix as a scaffold of tissue-engineered corneal epithelium. PLOS ONE 9, e111846 (2014)

160. Ma, X. Y. et al. Corneal stroma regeneration with acellular corneal stroma sheets and keratocytes in a rabbit model. PLOS ONE 10, e0132705 (2015)

161. Shafiq, M. A., Gemeinhart, R. A., Yue, B. \& Djalilian, A. R. Decellularized human cornea for reconstructing the corneal epithelium and anterior stroma. Tissue Eng. Part C Methods 18, 340-348 (2012)

162. Silva, J. M., Videira, M., Gaspar, R., Preat, V. \& Florindo, H. F. Immune system targeting by biodegradable nanoparticles for cancer vaccines. J. Control. Release 168, 179-199 (2013).

163. Hamdy, S., Haddadi, A., Hung, R. W. \& Lavasanifar, A. Targeting dendritic cells with nanoparticulate PLGA cancer vaccine formulations. Adv. Drug Deliv. Rev. 63, 943-955 (2011).

164. Kwong, B., Liu, H. \& Irvine, D. J. Induction of potent anti-tumor responses while eliminating systemic side effects via liposome-anchored combinatorial immunotherapy. Biomaterials 32, 5134-5147 (2011).

165. Moon, J. J., Huang, B. \& Irvine, D. J. Engineering nano- and microparticles to tune immunity. $A d v$. Mater. 24, 3724-3746 (2012)

166. Gammon, J. M., Tostanoski, L. H., Adapa, A. R. Chiu, Y.-C. \& Jewell, C. M. Controlled delivery of a metabolic modulator promotes regulatory $\mathrm{T}$ cells and restrains autoimmunity. J. Control. Release $\mathbf{2 1 0}$ 169-178 (2015).

167. Sunshine, J. C. \& Green, J. J. Nanoengineering approaches to the design of artificial antigenpresenting cells. Nanomedicine 8, 1173-1189 (2013).

168. Sunshine, J. C., Perica, K., Schneck, J. P. \& Green, J. J. Particle shape dependence of CD8 + $\mathrm{T}$ cell activation by artificial antigen presenting cells. Biomaterials 35, 269-277 (2014).

169. Ali, O. A. \& Mooney, D. J. in Cancer Immunology and Immunotherapy 279-297 (Springer, 2011).

170. Hori, Y., Winans, A. M., Huang, C. C., Horrigan, E. M. \& Irvine, D. J. Injectable dendritic cell-carrying alginate gels for immunization and immunotherapy. Biomaterials 29, 3671-3682 (2008)

171. Ali, O. A., Emerich, D., Dranoff, G. \& Mooney, D. J. In situ regulation of DC subsets and T cells mediates tumor regression in mice. Sci. Transl. Med. 1, 8ra19 (2009).

172. Bencherif, S. A. et al. Injectable cryogel-based whole-cell cancer vaccines. Nat. Commun. 6, 7556 (2015)

173. Vegas, A. J. et al. Combinatorial hydrogel library enables identification of materials that mitigate the foreign body response in primates. Nat. Biotechnol. 34, 345-352 (2016)
174. Ratner, B. D. Hoffman, A. S., Schoen, F. J. \& Lemons, J. E. (eds) Biomaterials Science 3rd edn 41-53 (Academic Press, 2013).

175. Kaul, H. \& Ventikos, Y. On the genealogy of tissue engineering and regenerative medicine. Tissue Eng. Part B, Rev. 21, 203-217 (2015).

176. Needham, J., Gwei-Djen, L. \& Sivin, N. Science and Civilisation in China: Volume 6, Biology and Biological Technology, Part 6, Medicine 134-135 (Cambridge University Press, 2000).

177. Jenner, E. On the Origin of the Vaccine Inoculation (G. Elsick, 1863)

178. Pasteur, L. \& Lister, J. Germ Theory and its Applications to Medicine and on the Antiseptic Principle of the Practice of Surgery (Prometheus Books, 1996).

179. Coley, W. B. The treatment of malignant tumors by repeated inoculations of erysipelas. With a report of ten original cases. 1893. Clin. Orthop. Relat. Res. 3-11 (1991).

180. Harris, W. H. \& Sledge, C. B. Total hip and total knee replacement. New Engl. J. Med. 323, 725-731 (1990)

181. Boon, T., Cerottini, J.-C., Van den Eynde, B., van der Bruggen, P. \& Van Pel, A. Tumor antigens recognized by T lymphocytes. Annu. Rev. Immunol. 12 337-365 (1994).

182. DiPietro, L. A. Wound healing: the role of the macrophage and other immune cells. Shock 4 233-240 (1995).

183. Barbul, A. Role of T-cell-dependent immune system in wound healing. Prog. Clin. Biol. Res. 266, 161-175 (1987).

184. Elisseeff, J. et al. Photoencapsulation of chondrocytes in poly(ethylene oxide)-based semiinterpenetrating networks. J. Biomed. Mater. Res. 51, 164-171 (2000)

185. Elisseeff, J. et al. Transdermal photopolymerization for minimally invasive implantation. Proc. Natl Acad. Sci. USA 96, 3104-3107 (1999).

186. Nicolson, P. C. $\&$ Vogt, J. Soft contact lens polymers an evolution. Biomaterials 22, 3273-3283 (2001).

187. Badylak, S. F. The extracellular matrix as a biologic scaffold material. Biomaterials 28, 3587-3593 (2007).

188. Badylak, S. F., Valentin, J. E., Ravindra, A. K., McCabe, G. P. \& Stewart-Akers, A. M. Macrophage phenotype as a determinant of biologic scaffold remodeling. Tissue Eng. Part A 14, 1835-1842 (2008).

189. Flannery, C. R. et al. Prevention of cartilage degeneration in a rat model of osteoarthritis by intraarticular treatment with recombinant lubricin. Arthritis Rheum. 60, 840-847 (2009)

190. Jones, A. R. et al. Binding and localization of recombinant lubricin to articular cartilage surfaces. J. Orthop. Res. 25, 283-292 (2007)

191. Kisiday, J. et al. Self-assembling peptide hydrogel fosters chondrocyte extracellular matrix production and cell division: implications for cartilage tissue repair. Proc. Natl Acad. Sci. USA 99, 9996-10001 (2002).

192. Sugiyama, H., Yamato, M., Nishida, K. \& Okano, T. Evidence of the survival of ectopically transplanted oral mucosal epithelial stem cells after repeated wounding of cornea. Mol. Ther. 22, 1544-1555 (2014).

193. Majumdar, S. et al. Influence of collagen source on fibrillar architecture and properties of vitrified collagen membranes. J. Biomed. Mater. Res. Part B 104B, 300-307 (2016)

194. Avadhanam, V. S., Smith, H. E. \& Liu, C. Keratoprostheses for corneal blindness: a review of contemporary devices. Clin. Ophthalmol. 9 697-720 (2015)

\section{Acknowledgements}

The authors acknowledge M. Frisk for helpful discussions and critical review, and the Bloomberg Kimmel Institute for Cancer Immunotherapy for support. M.T.W. was supported by a postdoctoral fellowship from the Hartwell Foundation. J.H.E was supported by the Jules and Doris Stein Research to Prevent Blindness Professorship. This work was funded by grants from the Armed Forces Institute of Regenerative Medicine (AFIRM), a US Department of Defense grant \#W81XWH-11-2-0022 and the Maryland Stem Cell Research Fund (MSCRF) \#113345 awarded to J.H.E.

Competing interests statement

The authors declare no competing interests. 